\title{
Regulated Salinity Eustress in a Floating Hydroponic Module of Sequentially Harvested Lettuce Modulates Phytochemical Constitution, Plant Resilience, and Post-Harvest Nutraceutical Quality
}

\author{
Petronia Carillo $^{1, *(\mathbb{D})}$, Georgios A. Soteriou ${ }^{2}{ }^{\mathbb{D}}$, Marios C. Kyriacou ${ }^{2}\left(\mathbb{D}\right.$, Maria Giordano $^{3} \mathbb{D}$, Giampaolo Raimondi $^{3}$, \\ Francesco Napolitano ${ }^{3}$, Emilio Di Stasio ${ }^{3}$, Ida Di Mola ${ }^{3}$, Mauro Mori ${ }^{3}$ and Youssef Rouphael ${ }^{3, *}$ (D) \\ 1 Department of Environmental, Biological and Pharmaceutical Sciences and Technologies, University of \\ Campania "Luigi Vanvitelli", Via Vivaldi 43, 81100 Caserta, Italy \\ 2 Department of Vegetable Crops, Agricultural Research Institute, Nicosia 1516, Cyprus; \\ soteriou@ari.gov.cy (G.A.S.); m.kyriacou@ari.gov.cy (M.C.K.) \\ 3 Department of Agricultural Sciences, University of Naples Federico II, 80055 Portici, Italy; \\ maria.giordano@unina.it (M.G.); giampaolo.raimondi@unina.it (G.R.); francesco.napolitano@hotmail.it (F.N.); \\ emiliodistasio@gmail.com (E.D.S.); ida.dimola@unina.it (I.D.M.); mori@unina.it (M.M.) \\ check for \\ * Correspondence: petronia.carillo@unicampania.it (P.C.); youssef.rouphael@unina.it (Y.R.)
} updates

Citation: Carillo, P.; Soteriou, G.A.; Kyriacou, M.C.; Giordano, M.; Raimondi, G.; Napolitano, F.; Di Stasio, E.; Mola, I.D.; Mori, M.; Rouphael, Y. Regulated Salinity Eustress in a Floating Hydroponic Module of Sequentially Harvested Lettuce Modulates Phytochemical Constitution, Plant Resilience, and Post-Harvest Nutraceutical Quality. Agronomy 2021, 11, 1040. https:// doi.org/10.3390/agronomy11061040

Academic Editor: Byoung

Ryong Jeong

Received: 29 March 2021

Accepted: 18 May 2021

Published: 22 May 2021

Publisher's Note: MDPI stays neutral with regard to jurisdictional claims in published maps and institutional affiliations.

Copyright: (C) 2021 by the authors. Licensee MDPI, Basel, Switzerland. This article is an open access article distributed under the terms and conditions of the Creative Commons Attribution (CC BY) license (https:// creativecommons.org/licenses/by/ $4.0 /)$

\begin{abstract}
A mild salinity stress (eustress) may modulate the induction of the plant defense system in horticultural crops and the synthesis of phytochemical components able to enhance plant resilience, post-harvest performance, and the nutraceutical quality of produce. However, the choice of the correct eustress type and dose to induce the synthesis of these protective phytochemicals is pivotal to avoid potential interference with plant growth and productivity. In order to study how green and red lettuce (Lactuca sativa L.) plants equilibrate the nutritional and nutraceutical components of quality with yield components, we applied iso-osmotic concentrations of three different salts $\left(20 \mathrm{mM} \mathrm{NaCl}, 20 \mathrm{mM} \mathrm{KCl}\right.$, and $13.3 \mathrm{mM} \mathrm{CaCl}_{2}$, with a final total ionic concentration of $40 \mathrm{mM}$ ) in combination with two successive harvests in a floating raft system. The biometric parameters, mineral composition, bioactive compounds, and antioxidant activity of both cultivars were analyzed. The green cultivar had a superior response concerning biometric traits and productivity compared to the red one during the first cut but lower phytochemical content (e.g., ascorbic acid). The effect of cut order, independently of cultivar and salinity treatments, demonstrated that at the first harvest plants could redirect metabolism by increasing the lipophilic antioxidant content (LAA) at the expense of plant yield, therefore increasing plant resilience and post-harvest nutraceutical quality; whereas, at the second harvest, plants reverted principally to tissue expansion. The treatments with iso-osmotic salt concentrations did not affect $\mathrm{K}$ and $\mathrm{Mg}$ ion contents but further increased LAA and resulted only in a moderate decrease of fresh yield. The lettuce nitrate content was reduced during the second cut only when lettuce plants were treated with $\mathrm{NaCl}$ and especially $\mathrm{CaCl}_{2}$.
\end{abstract}

Keywords: eustress; floating raft system; Lactuca sativa L.; iso-osmotic salts; phytochemicals; ions; yield

\section{Introduction}

The global leafy greens market size is expected to have a compound annual growth rate of $3.8 \%$ in the period 2020 to 2025, ranging from USD 643.1 million in 2019 to USD 746.8 million by 2025 [1]. These data clearly testify the increasing demand of consumers for health-promoting natural products and fresh foods. Green leafy vegetables, in particular, can provide minerals, dietary fibers, vitamins, and many other antioxidant metabolites essential for a balanced diet that reduces the risk of oxidative stress-related aging and degenerative diseases while promoting psychophysical well-being [2-5]. In Europe, the consumption of high quality ready-to-eat (RTE) 100\% edible leafy greens has reached 
a turnover of about 600 million Euros [6], and Italy ranked as the leader in Europe for the production of RTE leafy greens with about 15,000 ha and 160 kilotons of greenhouse production per year [7-9]. Among the most cultivated leafy greens worldwide for the minimally processed market, baby lettuce (Lactuca sativa L.) has the biggest success because of its optimal size (leaves with a petiole $\approx 8-12 \mathrm{~cm}$ long), low calorific value, and high nutraceutical content, in particular vitamins $C, E$, and $B_{9}$, and polyphenols [5,10-14]. The latter can play a dual role in prolonging the shelf-life of lettuce, reducing tissue decay and off-odours, and preventing cancer, cardiovascular, and neurodegenerative diseases [5,15-17]. The awareness of the functional properties of this horticultural product and the new eating habits of consumers have prompted researchers and producers to experiment with new agronomical practices, such as nutrient solution management and use of biostimulants for elicitation of maximal nutritional and nutraceutical quality in baby lettuce even under sub-optimal conditions [8,14,16,18-21]. In fact, a mild to moderate nutritional or salinity stress (eustress) may elicit plant defence responses by inducing the synthesis and accumulation of bioactive compounds [22-25], in particular of those able to detoxify reactive oxygen species (ROS) and promote plant hardening [8,18]. However, it is necessary to apply the right dose of stress/eustress so that the induction of the plant defence system and the consequent synthesis of protective metabolites do not occur at the expense of plant growth and productivity, achieving a fine-tuned equilibrium called sectio-divina $[8,21,26,27]$. Recent literature data prove that a salinity eustress at a dose of 5 and $30 \mathrm{mM} \mathrm{NaCl}$ could increase health-beneficial phenolic compounds in baby leaves [28], antioxidant metabolites such as total phenolics, luteolin, cynarine, and chlorogenic acid in leaves of artichoke and cardoon [29], and vitamin $C$ and $\alpha$-tocopherol contents in Cichorium spinosum [30], thus positively influencing post-harvest life and the nutritional quality of these products without altering plant yield. A eustress of $10 \mathrm{mM} \mathrm{NaCl}$ decreased the content of the antinutrient nitrate and enhanced that of polyphenols in both green and red-pigmented perilla, while a $20 \mathrm{mM} \mathrm{NaCl}$ eustress enhanced the content of compounds responsible for key aroma only in green perilla [27]. Lutein and $\beta$-carotene were strongly increased in cos (i.e., romaine) lettuce by $5 \mathrm{mM} \mathrm{NaCl}$ long-term irrigation without affecting yield or visual quality [18]. Neocleous, et al. [31] found that a mild salinity of $20 \mathrm{mM} \mathrm{NaCl}$ enhanced the anthocyanin content and coloration in red lettuce and improved freshness in green lettuce, but reduced leaf fresh weight. Similarly, the same concentration of $20 \mathrm{mM}$ $\mathrm{NaCl}$ increased beneficial minerals $(\mathrm{K}, \mathrm{Ca}$, and $\mathrm{Mg}$ ), lipophilic antioxidant activity, and vitamin $C$ in red lettuce, but not in green lettuce, causing a minimal loss of yield [8]. Moreover, an electrical conductivity of 3.8 and especially $4.8 \mathrm{mS} \mathrm{cm}^{-1}$ in the nutrient solution was able to enhance the properties of fresh-cut lettuce, reducing respiration, browning phenomena, the decay process, and prolonging its shelf life [32]. Therefore, while a mild salinity, $5 \mathrm{mM}<[\mathrm{NaCl}]<30 \mathrm{mM}$, can exert beneficial effects with a minimal impact on lettuce growth and biomass, high salinity concentrations $(\mathrm{NaCl}>30 \mathrm{mM})$ can limit plant growth and development by affecting ions and water uptake, thus causing oxidative stress with a consequent negative impact on photosynthesis, growth/yield, and quality [33]. Obviously, a low $\mathrm{NaCl}$ concentration $(\mathrm{NaCl} \sim 5 \mathrm{mM})$, does not affect growth and biomass and does not elicit a defence response [34].

Since even when using mild concentrations of salts it is not possible to discriminate between osmotic and ionic stress responses, it would be useful to examine the effect of isoosmotic solutions of salts on physiological and metabolic parameters of crop plants. They could exert a similar osmotic effect on plant metabolism, growth, and physiology, allowing us to unravel the response mechanisms of crop plants to salinity eustress. Notwithstanding that some studies on the use of iso-osmotic solutions of salts on crop plants have been done previously, the concentrations used cannot be defined as positive and/or beneficial. In fact, Mahmoudi, et al. [35] focused on the application of $100 \mathrm{mM} \mathrm{NaCl}$ and $77 \mathrm{mM} \mathrm{Na}_{2} \mathrm{SO}_{4}$ to Verte ( $\mathrm{NaCl}$ tolerant) and Romaine ( $\mathrm{NaCl}$ sensitive) lettuce, while Ntatsi, et al. [36] evaluated the effects of four sodium and chloride salts $\left(\mathrm{Na}_{2} \mathrm{SO}_{4}, \mathrm{NaCl}, \mathrm{KCl}\right.$ or $\left.\mathrm{CaCl}_{2}\right)$ on spiny chicory at final total ionic concentrations of $80 \mathrm{mM}$ and $160 \mathrm{mM}$. Ion-specific stress 
effects depend on the type of salt or its concentration and the salt-sensitivity of plants [37] and can induce beneficial or toxic effects depending on competition effects among ions or the predominance of specific ions [38]. In fact, the application of $\mathrm{CaCl}_{2}$ can have osmotic and ion specific effects stronger than those of $\mathrm{NaCl}$, reducing lettuce [39] and cucumber [40] yield, even at a concentration of $20 \mathrm{mM}$. This can be due to the toxic effects exerted by $\mathrm{Cl}^{-}$, whose uptake and transport to leaves seems to be less controlled than that of $\mathrm{Na}^{+}$, negatively affecting plant metabolism and development [40].

Successive harvests/cuts can modulate the quality of horticultural products, reshaping their final nutritional, nutraceutical, and sensory profile as well. In different basil genotypes, successive (two, three or four) cuts of the apical portion during the plant growth cycle could increase phenolic compounds and yield (as fresh biomass) [3,41]. Green and red lettuce at the second harvest showed higher dry biomass, mineral accumulation, and photosynthetic activity [8]. However, in spiny chicory, successive cuts increased total yield but, when more than two harvests were done, induced a decrease of sugars, tocopherols, and phenolic compounds and thus of antioxidant activity and quality of the produce [42].

Based on the above reasoning, finding the right eustress dose and type of salt to improve lettuce quality without compromising crop yield is of prime economic importance. In addition, the comprehension of the effects of iso-osmotic salts may provide a tool to fine-tune the nutritional and nutraceutical profiles of lettuce plants [43]. Therefore, our study aimed to examine how mild iso-osmotic concentrations of three different salts $\left(20 \mathrm{mM} \mathrm{NaCl}, 20 \mathrm{mM} \mathrm{KCl}\right.$, and $13.3 \mathrm{mM} \mathrm{CaCl}_{2}$, with a final total ionic concentration of $40 \mathrm{mM}$ ) interacted with successive harvests to modulate the physical, physiological, and biochemical parameters in green- and red-pigmented lettuce. These treatments allowed studying not only the possible positive ionic effects induced by mild amounts of $\mathrm{Na}$ and $\mathrm{Cl}$, or the beneficial effects of $\mathrm{K}$ and $\mathrm{Ca}$, completely excluding the effects related to osmotic stress, but also the effects of successive harvests.

\section{Materials and Methods}

\subsection{Lettuce Cultivars, Greenhouse Conditions, and Experimental Design}

This work was conducted on two minimally processed Lactuca sativa L. var. acephala cultivars: Green and Red Salad Bowl (Società Agricola Italiana Sementi, S.A.I.S s.p.a., Cesena, Italy) characterized by green and red pigmentation, respectively. These cultivars were therefore named Green and Red throughout the manuscript.

The experiment was carried out in the 2018 spring growing season (from 21 March to 4 May), in an unheated glasshouse (10 m wide, $30 \mathrm{~m}$ long, 3 and $4.5 \mathrm{~m}$ high at the eaves and ridge, respectively) at the pilot farm "Torre Lama" (Bellizzi, Salerno, Italy) of the Department of Agricultural Sciences (University Federico II of Naples). The red and green-pigmented Lactuca sativa L. var. acephala were sown in 180-hole $(60 \mathrm{~cm} \times 30 \mathrm{~cm})$ polystyrene trays with a planting density of 1149 plants $\mathrm{m}^{-2}$. Eight days after sowing (DAS), seedlings of the two baby lettuce cultivars were moved to a floating system. The floating raft system consisted of polystyrene polystyrene-trays, floating in tanks with a constant volume (150 L) of fresh nutrient solution (NS). In each experimental unit, an immersion pump was placed to maintain a constant dissolved oxygen $\left(\mathrm{O}_{2}\right)$ level above the threshold limit of $6 \mathrm{mg} \mathrm{L}^{-1}$.

The experimental design adopted in this work was a full factorial with three experimental factors (F): Cultivars (CV: Green and Red), Salinity sources (S: non saline control and iso-osmotic concentrations of three salts $\left.\mathrm{NaCl}, \mathrm{KCl}, \mathrm{CaCl}_{2}\right)$, and successive harvest $(\mathrm{C}$ : cut 1 and cut 2). Treatments were arranged in a randomized complete block design with three replicates $(\mathrm{R})$.

\subsection{Nutrient Solution Composition and Iso-Osmotic Salt Application}

The macro $(\mathrm{mM})$ and micronutrients $(\mu \mathrm{M})$ concentrations of the basic NS (i.e., nonsaline control) were: $\mathrm{N}^{-N_{3}}{ }^{-}: 13.0 ; \mathrm{N}^{-N_{4}}{ }_{4}^{+}$: $1.0 ; \mathrm{S}: 1.75 ; \mathrm{P}: 1.5 ; \mathrm{K}: 5.0 ; \mathrm{Ca}$ : 4.5; Mg: 2.0; Fe: 20; $\mathrm{Mn}: 9 ; \mathrm{Cu}: 0.3 ; \mathrm{Zn}: 1.6 ; \mathrm{B}: 20 ; \mathrm{Mo}$ : 0.3. The electrical conductivity (EC) and $\mathrm{pH}$ of the 
basic NS were $2.0 \mathrm{dS} \mathrm{m}^{-1}$ and 6.0, respectively. In addition to the non-saline control NS, three iso-osmotic saline NSs were obtained by adding to the basic NS different amounts of sodium chloride $(20 \mathrm{mM} \mathrm{NaCl})$, potassium chloride $(20 \mathrm{mM} \mathrm{KCl})$, and calcium chloride $\left(13.3 \mathrm{mM} \mathrm{CaCl}_{2}\right)$. The salt concentration in the three different NSs were iso-osmotic in order to achieve the same total ionic concentration of $40 \mathrm{mM}$. The NS in all treatments was replaced weekly to guarantee the same initial mineral nutrient conditions.

\subsection{Sampling, Growth, Yield, and Leaf Biomass Determination}

The two minimally processed Lactuca sativa L. var. acephala cultivars were harvested when the control treatment reached the commercial stage, 25 and 29 DAS for green and redpigmented baby lettuce, for the first harvest (Cut 1). The second harvest (Cut 2) occurred 43 and 45 DAS, for the green and red cultivar, respectively.

At each harvest date, the fresh weight (fw; expressed as $\mathrm{kg}$ per square meter) of both green and red-pigmented lettuce was recorded. Consecutively, the total leaf area expressed as $\mathrm{cm}^{2}$ per plant was measured using an automatic LiCor 3100C area meter (LI-COR Biosciences, Lincoln, NE, USA). A subsample of each plant was stored in paper bags and dried in a forced-air oven at $70{ }^{\circ} \mathrm{C}$ until constant weight $(72 \mathrm{~h})$ to determine the dry weight $(\mathrm{dw})$. Dry matter content was calculated as follows: $\mathrm{dw} / \mathrm{fw} \times 100$. A sample of plants was collected and immediately frozen in liquid nitrogen and stored at $-80^{\circ} \mathrm{C}$ before being freeze-dried for further qualitative analysis (i.e., bioactive molecules and antioxidant activities). For mineral composition, the dry lettuce material was milled and sieved with a MF10.1 Wiley laboratory mill equipped with a MF0.5 sieve (IKA, Staufen im Breisgau, Baden-Württemberg, Germany).

\subsection{CIELAB Leaf Colorimetry and Quality Analysis}

Twenty colorimetric coordinates were recorded on twenty representative red/green leaves of each experimental unit at each harvest date, using a Chroma Meter Minolta CR-300 (Minolta Co. Ltd., Osaka, Japan) calibrated with a correspondent Minolta standard. The color spaces were expressed with $\mathrm{L}^{*}, \mathrm{a}^{*}$, and $\mathrm{b}^{*}$ values.

The fresh lettuce leaves were homogenized in a blender (2 L capacity; Waring HGB140, CA, USA) for one minute at low speed. The slurry was filtered through a two-layer cheesecloth, and the total soluble solids (TSS; ${ }^{\circ} \mathrm{Brix}$ ) content, juice EC, and $\mathrm{pH}$ were read with a digital Atago N1 refractometer (Tokyo, Japan), HI-9023 pH meter, and HI-99301 EC meter (Hanna Instruments, Padova, Italy), respectively.

\subsection{Macronutrients, Sodium and Chloride Determination}

Dried and ground baby lettuce leaf tissues were processed for total nitrogen $(\mathrm{N})$, macronutrients $(\mathrm{P}, \mathrm{K}, \mathrm{Ca}$ and $\mathrm{Mg})$, sodium $(\mathrm{Na})$, and chlorine $(\mathrm{Cl})$ analysis. The total $\mathrm{N}$ was determined by the Kjeldahl method [44]. The ion chromatography system ICS 3000 (Thermo Scientific Dionex, Sunnyvale, CA, USA) was used to determine the macronutrient, $\mathrm{Na}$, and $\mathrm{Cl}$ concentrations of baby lettuce, following the protocol described by Rouphael and co-workers [45]. For the determination of the cations (K-Ca-Mg-Na), the IonPac CG12A guard column $(4 \times 250 \mathrm{~mm})$ and the IonPac CS12A analytical column $(4 \times 250 \mathrm{~mm})$ were used, whereas the IonPac AG11-HC guard column $(4 \times 50 \mathrm{~mm})$ and the IonPac AS11-HC analytical column $(4 \times 250 \mathrm{~mm})$ were used for anion $(\mathrm{P}-\mathrm{Cl})$ determination. The ion concentrations of the tested samples were calculated based on the standard curves of cations and anions. All chemicals were purchased from Sigma Aldrich (Milan, Italy).

\subsection{Bioactive Molecules and Antioxidant Activities Quantification}

Determinations of the lipophilic (LAA) and hydrophilic (HAA) antioxidant activity were evaluated using lyophilized leaf tissues, whereas total ascorbic acid was done on fresh biomass using liquid nitrogen. The ultraviolet-visible (UV-Vis) spectrophotometer Hach DR 2000 (Hach Co., Loveland, CO, USA) was used to quantify the total ascorbic acid (TAA) and the lipophilic antioxidant activity (LAA) and the hydrophilic antioxidant activity 
(HAA). TAA was assessed following the protocol described by Kampfenkel, et al. [46], whereas the LAA and HAA were determined following the ABTS and DMPD methods described by Re, et al. [47] and Fogliano, et al. [48], respectively. The sample absorbance was read at 525, 734, and $505 \mathrm{~nm}$ for TAA, LAA and HAA respectively.

\subsection{Statistics}

Three-way analysis of variance (ANOVA) was implemented to assess the significance of the effects and interaction between the tested factors: Cultivar-CV, Salinity-S, and Cut-C. One-way ANOVA was used to compare the mean effect of the three tested factors $(\mathrm{CV}, \mathrm{S}$, and C) according to Duncan's Multiple Range Test (DRMT). Moreover, statistical significance was determined at the $p<0.05$ level using DRMT for $\mathrm{CV} \times \mathrm{S}, \mathrm{S} \times \mathrm{C}$, and $\mathrm{CV} \times \mathrm{C}$ interactions. All data are presented as the mean \pm standard error. All statistical analyses were performed using the IBM SPSS 20 (Armonkn, NY, USA) package for Microsoft Windows 10. The principal component analysis (PCA) was performed using Minitab 18 statistical software (Minitab LLC, State College, PA, USA).

\section{Results}

\subsection{Biometric Traits and Productivity Response}

Most of the biometric traits were affected by the type of cultivar and the successive cuts, but only fresh yield was affected by salinity (Table 1). Significant differences were observed between baby lettuce cultivars since green lettuce produced, in almost all cuts, superior leaf area and fresh yield than the red cultivar (Table 1). No cultivar differentiation was observed for leaf dry matter (DM). Nonetheless, a significant interaction between cultivar and cut was observed for leaf area and fresh yield as the two lettuce cultivars behaved dissimilarly between the two cuts. Further statistical analysis of the leaf area and fresh yield $\mathrm{CV} \times \mathrm{C}$ significant interaction revealed that the leaf area and fresh yield of green lettuce remained the same during the two cuts and were superior to those of red lettuce only during the first cut. Both cultivars' dry biomass and leaf dry matter increased during the second cut, but the increase of the red cultivar was greater. Finally, while salinity source treatments had no effect on the leaf area, dry biomass, and leaf DM percentage, $\mathrm{KCl}$ and $\mathrm{NaCl}$ treatments suppressed (by 18.1\%) the fresh marketable yield. Interestingly, the non-saline control and $\mathrm{CaCl}_{2}$ treatments did not differ significantly from each other (Table 1).

\subsection{Leaf Colorimetry and Flavor Compounds}

The leaf colorimetric attributes examined $\left(\mathrm{L}^{*}, \mathrm{a}^{*}\right.$, and $\left.\mathrm{b}^{*}\right)$, were mostly affected by the cultivar type and to a lesser extent by cut order since they were largely reflected by the obvious genotypic color differences of the cultivars assessed (green versus red-pigmented lettuce) (Table 2). The deepest coloration resulted from more intense redness (higher $\mathrm{a}^{*}$ values), and lower lightness (lower $L^{*}$ values) and yellowness (lower $b^{*}$ values) were observed in red-pigmented baby lettuce, whereas the lightest coloration (lowest redness and highest lightness and yellowness) was observed in the green counterpart (Table 2). The color of both cultivars increased in intensity during the second cut due to the reduction of the $b^{*}$ value.

No cultivar or salinity treatment differentiation was observed for the TSS content. The TSS content increased by $28.9 \%$, on average, during the successive cuts (Table 2). Juice EC was also not affected by cultivar, but an opposite trend to TSS was recorded during the two cuts. A significant $S \times C$ interaction was observed concerning juice EC, as salinity treatments influenced lettuce plant EC only during the second cut. Juice pH was affected only by the salinity treatments. However, a significant $\mathrm{S} \times \mathrm{C}$ interaction was observed as lettuce plants treated with $\mathrm{KCl}$ or $\mathrm{NaCl}$ exhibited higher juice $\mathrm{pH}$ values, only during the second cut, compared to the $\mathrm{CaCl}_{2}$ treatment. 
Table 1. Significance of the main factors (cultivar, salinity, and cut order) and their interactions on the leaf area, fresh yield, dry biomass, and leaf dry matter percentage of Lactuca sativa L. var. acephala grown in a floating system.

\begin{tabular}{|c|c|c|c|c|}
\hline Source of Variance & $\begin{array}{c}\text { Leaf Area } \\
\left(\mathrm{cm}^{2} \text { Plant }^{-1}\right)\end{array}$ & $\begin{array}{l}\text { Fresh Yield } \\
\left(\mathrm{kg} \mathrm{m}^{-2}\right)\end{array}$ & $\begin{array}{l}\text { Dry Biomass } \\
\qquad\left(\mathrm{g} \mathrm{m}^{-2}\right)\end{array}$ & $\begin{array}{c}\text { Leaf Dry Matter } \\
(\%)\end{array}$ \\
\hline \multicolumn{5}{|l|}{ Cultivar (CV) } \\
\hline Green & $99.39 \pm 2.36 \mathrm{a}$ & $4.29 \pm 0.13 \mathrm{a}$ & $218.17 \pm 9.56 \mathrm{a}$ & $5.11 \pm 0.15$ \\
\hline Red & $90.79 \pm 3.56 b$ & $3.65 \pm 0.16 b$ & $177.16 \pm 10.72 b$ & $4.80 \pm 0.14$ \\
\hline \multicolumn{5}{|l|}{ Salinity (S) } \\
\hline Control & $102.27 \pm 4.77$ & $4.48 \pm 0.25 \mathrm{a}$ & $221.89 \pm 16.41$ & $4.98 \pm 0.19$ \\
\hline $\mathrm{CaCl} 2$ & $96.38 \pm 4.66$ & $4.06 \pm 0.23 \mathrm{ab}$ & $204.70 \pm 17.36$ & $4.99 \pm 0.23$ \\
\hline $\mathrm{KCl}$ & $90.04 \pm 4.62$ & $3.66 \pm 0.20 \mathrm{~b}$ & $180.82 \pm 14.52$ & $4.88 \pm 0.18$ \\
\hline $\mathrm{NaCl}$ & $91.66 \pm 3$ & $3.68 \pm 0.15 b$ & $183.25 \pm 11.48$ & $4.98 \pm 0.24$ \\
\hline \multicolumn{5}{|l|}{ Cut (C) } \\
\hline Cut 1 & $89.14 \pm 2.93 b$ & $3.78 \pm 0.17 \mathrm{~b}$ & $170.42 \pm 8.00 b$ & $4.53 \pm 0.06 \mathrm{~b}$ \\
\hline $\begin{array}{l}\text { Cut } 2 \\
C V \times S\end{array}$ & $101.05 \pm 2.87 \mathrm{a}$ & $4.16 \pm 0.14 \mathrm{a}$ & $224.91 \pm 10.69 \mathrm{a}$ & $5.38 \pm 0.15 a$ \\
\hline Control, Green & $103.28 \pm 6.26$ & $4.70 \pm 0.35$ & $239.69 \pm 21.67$ & $5.21 \pm 0.27$ \\
\hline Control, Red & $101.27 \pm 7.78$ & $4.27 \pm 0.37$ & $204.08 \pm 24.26$ & $4.74 \pm 0.24$ \\
\hline $\mathrm{CaCl} 2$, Green & $104.33 \pm 3.89$ & $4.53 \pm 0.21$ & $242.46 \pm 19.54$ & $5.36 \pm 0.36$ \\
\hline $\mathrm{CaCl} 2, \mathrm{Red}$ & $88.43 \pm 7.43$ & $3.60 \pm 0.32$ & $166.94 \pm 19.33$ & $4.62 \pm 0.25$ \\
\hline $\mathrm{KCl}$, Green & $95.1 \pm 4.95$ & $4.01 \pm 0.21$ & $197.67 \pm 16.59$ & $4.91 \pm 0.24$ \\
\hline $\mathrm{KCl}$, Red & $84.99 \pm 7.69$ & $3.30 \pm 0.29$ & $163.98 \pm 23.22$ & $4.85 \pm 0.28$ \\
\hline $\mathrm{NaCl}$, Green & $94.84 \pm 2.75$ & $3.92 \pm 0.19$ & $192.87 \pm 11.55$ & $4.96 \pm 0.32$ \\
\hline $\begin{array}{l}\mathrm{NaCl}, \mathrm{Red} \\
\mathrm{S} \times \mathrm{C}\end{array}$ & $88.49 \pm 5.29$ & $3.44 \pm 0.21$ & $173.63 \pm 20.22$ & $5.00 \pm 0.38$ \\
\hline Control, Cut 1 & $90.71 \pm 4.14$ & $4.23 \pm 0.40$ & $192.98 \pm 15.67$ & $4.70 \pm 0.13$ \\
\hline Control, Cut 2 & $113.84 \pm 5.43$ & $4.74 \pm 0.31$ & $250.79 \pm 24.6$ & $5.26 \pm 0.32$ \\
\hline $\mathrm{CaCl} 2$, Cut 1 & $88.88 \pm 7.86$ & $3.77 \pm 0.41$ & $172.61 \pm 18.71$ & $4.58 \pm 0.08$ \\
\hline $\mathrm{CaCl} 2, \mathrm{Cut} 2$ & $103.88 \pm 3.36$ & $4.36 \pm 0.17$ & $236.79 \pm 23.75$ & $5.40 \pm 0.41$ \\
\hline $\mathrm{KCl}$, Cut 1 & $86.85 \pm 7.96$ & $3.46 \pm 0.31$ & $156.48 \pm 17.93$ & $4.47 \pm 0.15$ \\
\hline $\mathrm{KCl}$, Cut 2 & $93.24 \pm 5.14$ & $3.85 \pm 0.25$ & $205.17 \pm 19.23$ & $5.29 \pm 0.22$ \\
\hline $\mathrm{NaCl}$, Cut 1 & $90.11 \pm 3.65$ & $3.65 \pm 0.21$ & $159.63 \pm 9.82$ & $4.38 \pm 0.12$ \\
\hline $\begin{array}{c}\mathrm{NaCl}, \mathrm{Cut} 2 \\
\mathrm{CV} \times \mathrm{C}\end{array}$ & $93.22 \pm 5.04$ & $3.71 \pm 0.25$ & $206.88 \pm 16.12$ & $5.58 \pm 0.29$ \\
\hline Green, Cut 1 & $97.79 \pm 3.15 \mathrm{a}$ & $4.37 \pm 0.20 \mathrm{a}$ & $197.24 \pm 8.93$ & $4.58 \pm 0.10$ \\
\hline Green, Cut 2 & $100.98 \pm 3.59 \mathrm{a}$ & $4.21 \pm 0.19 \mathrm{a}$ & $239.1 \pm 14.93$ & $5.65 \pm 0.16$ \\
\hline Red, Cut 1 & $80.48 \pm 3.5 b$ & $3.18 \pm 0.13 b$ & $143.61 \pm 7.56$ & $4.49 \pm 0.08$ \\
\hline Red, Cut 2 & $101.11 \pm 4.64 \mathrm{a}$ & $4.12 \pm 0.23 \mathrm{a}$ & $210.71 \pm 14.78$ & $5.11 \pm 0.24$ \\
\hline Significance & & & & \\
\hline Cultivar (CV) & * & $* *$ & $* *$ & n.s. \\
\hline Salinity (S) & n.s. & $* *$ & n.s. & n.s. \\
\hline Cut (C) & $* *$ & * & $* * *$ & $* * *$ \\
\hline $\mathrm{CV} \times \mathrm{S}$ & n.s. & n.s. & n.s. & n.s. \\
\hline $\mathrm{S} \times \mathrm{C}$ & n.s. & n.s. & n.s. & n.s. \\
\hline $\mathrm{CV} \times \mathrm{C}$ & * & $* *$ & n.s. & n.s. \\
\hline $\mathrm{CV} \times \mathrm{S} \times \mathrm{C}$ & n.s. & n.s. & n.s. & n.s. \\
\hline
\end{tabular}

n.s., ${ }^{* * *}, * * *$ Not significant or significant at $p \leq 0.05,0.01$, and 0.001 , respectively. Different letters indicate statistically different groups ( $p<0.05$, Duncan's post hoc test following ANOVA; $\mathrm{n}=3$ ). 
Table 2. Significance of the main factors (cultivar, salinity, and cut order) and their interactions on leaf colorimetric attributes $\left(\mathrm{L}^{*}, \mathrm{a}^{*}\right.$, and $\left.\mathrm{b}^{*}\right)$, total soluble solids (TSS; ${ }^{\circ}$ Brix) content, juice $\mathrm{pH}$, and juice electrical conductivity $\left(\mathrm{EC} ; \mathrm{dSm}^{-1}\right)$ of Lactuca sativa L. var. acephala grown in a floating system.

\begin{tabular}{|c|c|c|c|c|c|c|}
\hline Source of Variance & $\mathbf{L}^{*}$ & $a^{*}$ & $\mathbf{b}^{*}$ & TSS & Juice $\mathrm{pH}$ & Juice EC \\
\hline \multicolumn{7}{|l|}{ Cultivar (CV) } \\
\hline Green & $50.28 \pm 0.39 a$ & $-7.84 \pm 0.15 b$ & $24.58 \pm 0.49 \mathrm{a}$ & $4.75 \pm 0.22$ & $5.99 \pm 0.02$ & $4.89 \pm 0.19$ \\
\hline Red & $41.26 \pm 0.56 b$ & $-4.09 \pm 0.33 \mathrm{a}$ & $16.42 \pm 0.55 b$ & $4.82 \pm 0.20$ & $6.03 \pm 0.02$ & $4.73 \pm 0.24$ \\
\hline \multicolumn{7}{|l|}{ Salinity (S) } \\
\hline Control & $45.40 \pm 1.90$ & $-6.09 \pm 0.88$ & $20.58 \pm 1.81$ & $4.37 \pm 0.18$ & $5.99 \pm 0.02 \mathrm{ab}$ & $4.25 \pm 0.25 b$ \\
\hline $\mathrm{CaCl} 2$ & $45.11 \pm 1.40$ & $-6.52 \pm 0.55$ & $20.51 \pm 1.40$ & $4.68 \pm 0.28$ & $5.93 \pm 0.02 b$ & $5.13 \pm 0.28 \mathrm{a}$ \\
\hline $\mathrm{KCl}$ & $45.78 \pm 1.58$ & $-5.43 \pm 0.73$ & $19.65 \pm 1.42$ & $5.06 \pm 0.30$ & $6.05 \pm 0.03 \mathrm{a}$ & $4.87 \pm 0.36 \mathrm{ab}$ \\
\hline $\mathrm{NaCl}$ & $46.79 \pm 1.04$ & $-6.37 \pm 0.50$ & $21.26 \pm 0.98$ & $5.03 \pm 0.38$ & $6.07 \pm 0.03 \mathrm{a}$ & $4.99 \pm 0.28 \mathrm{a}$ \\
\hline \multicolumn{7}{|l|}{ Cut (C) } \\
\hline Cut 1 & $45.91 \pm 1.00$ & $-6.32 \pm 0.44$ & $21.45 \pm 0.95 \mathrm{a}$ & $4.18 \pm 0.12 b$ & $6.00 \pm 0.02$ & $5.11 \pm 0.15 a$ \\
\hline Cut 2 & $45.63 \pm 1.11$ & $-5.85 \pm 0.50$ & $19.55 \pm 1.00 \mathrm{~b}$ & $5.39 \pm 0.21 \mathrm{a}$ & $6.02 \pm 0.02$ & $4.51 \pm 0.25 b$ \\
\hline \multicolumn{7}{|l|}{$\mathrm{CV} \times \mathrm{S}$} \\
\hline Control, Green & $51.50 \pm 0.94$ & $-8.12 \pm 0.49$ & $25.76 \pm 1.70$ & $4.40 \pm 0.21$ & $5.97 \pm 0.02$ & $4.54 \pm 0.26$ \\
\hline Control, Red & $39.31 \pm 0.45$ & $-3.04 \pm 0.19$ & $15.39 \pm 0.85$ & $4.33 \pm 0.30$ & $6.02 \pm 0.03$ & $3.96 \pm 0.42$ \\
\hline $\mathrm{CaCl} 2$,Green & $49.36 \pm 0.51$ & $-8.02 \pm 0.13$ & $24.72 \pm 0.31$ & $4.65 \pm 0.41$ & $5.90 \pm 0.03$ & $4.81 \pm 0.49$ \\
\hline $\mathrm{CaCl} 2, \mathrm{Red}$ & $40.86 \pm 1.07$ & $-4.72 \pm 0.43$ & $16.29 \pm 1.21$ & $4.72 \pm 0.41$ & $5.96 \pm 0.02$ & $5.45 \pm 0.25$ \\
\hline $\mathrm{KCl}$, Green & $50.34 \pm 0.89$ & $-7.52 \pm 0.33$ & $23.72 \pm 0.83$ & $5.08 \pm 0.56$ & $6.07 \pm 0.05$ & $5.07 \pm 0.51$ \\
\hline $\mathrm{KCl}$, Red & $41.22 \pm 1.37$ & $-3.33 \pm 0.71$ & $15.58 \pm 1.28$ & $5.03 \pm 0.30$ & $6.04 \pm 0.04$ & $4.66 \pm 0.54$ \\
\hline $\mathrm{NaCl}$, Green & $49.93 \pm 0.56$ & $-7.69 \pm 0.17$ & $24.12 \pm 0.57$ & $4.87 \pm 0.59$ & $6.03 \pm 0.03$ & $5.14 \pm 0.26$ \\
\hline $\begin{array}{c}\mathrm{NaCl}, \mathrm{Red} \\
\mathrm{S} \times \mathrm{C}\end{array}$ & $43.66 \pm 0.73$ & $-5.04 \pm 0.59$ & $18.41 \pm 0.77$ & $5.18 \pm 0.54$ & $6.10 \pm 0.05$ & $4.84 \pm 0.51$ \\
\hline Control, Cut 1 & $44.97 \pm 2.98$ & $-6.63 \pm 1.47$ & $22.65 \pm 2.90$ & $4.20 \pm 0.29$ & $6.03 \pm 0.03 \mathrm{abc}$ & $4.82 \pm 0.21 \mathrm{ab}$ \\
\hline Control, Cut 2 & $45.83 \pm 2.64$ & $-5.55 \pm 1.09$ & $18.50 \pm 2.06$ & $4.53 \pm 0.21$ & $5.96 \pm 0.02 b c$ & $3.69 \pm 0.33 b$ \\
\hline $\mathrm{CaCl} 2, \mathrm{Cut} 1$ & $45.80 \pm 1.81$ & $-6.61 \pm 0.73$ & $21.56 \pm 1.64$ & $3.93 \pm 0.24$ & $5.93 \pm 0.03 c$ & $4.57 \pm 0.36 \mathrm{ab}$ \\
\hline $\mathrm{CaCl} 2$, Cut 2 & $44.42 \pm 2.27$ & $-6.40 \pm 0.94$ & $19.46 \pm 2.35$ & $5.44 \pm 0.24$ & $5.93 \pm 0.02 c$ & $5.68 \pm 0.30 \mathrm{a}$ \\
\hline $\mathrm{KCl}$, Cut 1 & $45.57 \pm 1.70$ & $-5.47 \pm 0.83$ & $19.74 \pm 1.63$ & $4.37 \pm 0.22$ & $5.99 \pm 0.04 \mathrm{abc}$ & $5.62 \pm 0.22 \mathrm{a}$ \\
\hline $\mathrm{KCl}, \mathrm{Cut} 2$ & $45.99 \pm 2.84$ & $-5.39 \pm 1.30$ & $19.56 \pm 2.50$ & $5.75 \pm 0.39$ & $6.12 \pm 0.03 a$ & $4.12 \pm 0.54 b$ \\
\hline $\mathrm{NaCl}, \mathrm{Cut} 1$ & $47.29 \pm 1.64$ & $-6.61 \pm 0.63$ & $21.87 \pm 1.44$ & $4.20 \pm 0.27$ & $6.05 \pm 0.05 \mathrm{abc}$ & $5.43 \pm 0.25 \mathrm{a}$ \\
\hline $\begin{array}{c}\mathrm{NaCl}, \text { Cut } 2 \\
\mathrm{CV} \times \mathrm{C}\end{array}$ & $46.29 \pm 1.41$ & $-6.12 \pm 0.81$ & $20.66 \pm 1.40$ & $5.85 \pm 0.55$ & $6.08 \pm 0.02 \mathrm{ab}$ & $4.55 \pm 0.45 \mathrm{ab}$ \\
\hline Green, Cut 1 & $50.24 \pm 0.62$ & $-8.02 \pm 0.28$ & $25.41 \pm 0.89$ & $3.98 \pm 0.11$ & $5.96 \pm 0.02$ & $4.93 \pm 0.25$ \\
\hline Green, Cut 2 & $50.32 \pm 0.49$ & $-7.66 \pm 0.13$ & $23.76 \pm 0.33$ & $5.53 \pm 0.30$ & $6.03 \pm 0.03$ & $4.85 \pm 0.30$ \\
\hline Red, Cut 1 & $41.58 \pm 0.62$ & $-4.46 \pm 0.37$ & $17.50 \pm 0.42$ & $4.38 \pm 0.21$ & $6.05 \pm 0.03$ & $5.29 \pm 0.17$ \\
\hline Red, Cut 2 & $40.94 \pm 0.95$ & $-3.69 \pm 0.55$ & $15.34 \pm 0.93$ & $5.26 \pm 0.29$ & $6.01 \pm 0.02$ & $4.16 \pm 0.38$ \\
\hline \multicolumn{7}{|l|}{ Significance } \\
\hline Cultivar (CV) & $* * *$ & $* * *$ & $* * *$ & n.s. & n.s. & n.s. \\
\hline Salinity (S) & n.s. & n.s. & n.s. & n.s. & $* * *$ & $* *$ \\
\hline Cut (C) & n.s. & n.s. & $* *$ & $* * *$ & n.s. & * \\
\hline $\mathrm{CV} \times \mathrm{S}$ & n.s. & n.s. & n.s. & n.s. & n.s. & n.s. \\
\hline $\mathrm{S} \times \mathrm{C}$ & n.s. & n.s. & n.s. & n.s. & $*$ & $* *$ \\
\hline $\mathrm{CV} \times \mathrm{C}$ & n.s. & n.s. & n.s. & n.s. & n.s. & n.s. \\
\hline $\mathrm{CV} \times \mathrm{S} \times \mathrm{C}$ & n.s. & n.s. & n.s. & n.s. & n.s. & n.s. \\
\hline
\end{tabular}

n.s., ${ }^{*}, * * * * *$ Non-significant or significant at $p \leq 0.05,0.01$, and 0.001 , respectively. Different letters indicate statistically different groups $(p<0.05$, Duncan's post hoc test following ANOVA; $\mathrm{n}=3)$.

\subsection{Leaf Mineral Profile, $\mathrm{Na}$ and $\mathrm{Cl}$ Accumulation}

The salinity treatments had the most pronounced effect on the leaf content for all minerals analyzed followed by the two other tested factors: cultivar and cut order (Table 3). Cut order was the only factor with a significant effect on the leaf $\mathrm{N}$ content, which was higher in the first than in the second harvest (Table 3). Contrary to total N, the P content was higher in the second than in the first harvest ( 3.3 versus $2.8 \mathrm{~g} \mathrm{~kg}^{-1} \mathrm{dw}$ ). The salinity factor also had a significant effect on the $\mathrm{P}$ content which decreased in response to the $\mathrm{CaCl}_{2}$ treatment (Table 3 ). The potassium content was only affected by salinity and not by cultivar and cut order; it was higher in lettuce plants fertigated with $20 \mathrm{mM} \mathrm{KCl}$ (Table 3). 
The contents of the two bivalent cations, $\mathrm{Ca}$ and $\mathrm{Mg}$, were affected by all the experimental factors examined and were higher by $34.7 \%$ and $20.8 \%$ (for $\mathrm{Ca}$ and $\mathrm{Mg}$, respectively) in the green lettuce compared to the red one, and almost doubled at cut 2 compared to cut 1 (Table 3). However, further analysis of the $\mathrm{S} \times \mathrm{C}$ interaction for the Ca content demonstrated that it was increased during the second cut only in plants grown in the absence of salt or treated with $\mathrm{CaCl}_{2}$. Moreover, the $\mathrm{KCl}$ and $\mathrm{NaCl}$ treatments decreased the $\mathrm{Mg}$ concentration in leaf tissue, while the non-saline control and $\mathrm{CaCl}_{2}$ treatments did not differ significantly from each other (Table 3).

Table 3. Significance of the main factors (cultivar, salinity, and cut order) and their interactions on leaf mineral composition (total nitrogen $[\mathrm{N}]$, phosphorus [P], potassium [K], calcium [Ca], magnesium [Mg], sodium [Na], chloride [Cl]) of Lactuca sativa L. var. acephala grown in a floating system.

\begin{tabular}{|c|c|c|c|c|c|c|c|}
\hline Source of Variance & $\frac{N}{\left(g_{k g}^{-1} \mathrm{dw}\right)}$ & $\underset{\left(\mathrm{g} \mathrm{kg}^{-1} \mathrm{dw}\right)}{P}$ & $\frac{\mathrm{K}}{\left(\mathrm{g} \mathrm{kg}^{-1} \mathrm{dw}\right)}$ & $\frac{\text { Ca }}{\left(\mathrm{g} \mathrm{kg}^{-1} \mathrm{dw}\right)}$ & $\underset{\left(\mathrm{g} \mathrm{kg}^{-1} \mathrm{dw}\right)}{\mathrm{Mg}}$ & $\underset{\left(\mathrm{g} \mathrm{kg}^{-1} \mathrm{dw}\right)}{\mathrm{Na}}$ & $\frac{\mathrm{Cl}}{\left(\mathrm{g} \mathrm{kg}^{-1} \mathrm{dw}\right)}$ \\
\hline \multicolumn{8}{|l|}{ Cultivar (CV) } \\
\hline Green & $44.92 \pm 0.46$ & $3.14 \pm 0.09$ & $50.51 \pm 2.99$ & $7.36 \pm 0.77 \mathrm{a}$ & $2.09 \pm 0.11 \mathrm{a}$ & $5.66 \pm 1.32 \mathrm{a}$ & $23.46 \pm 2.85$ \\
\hline Red & $43.94 \pm 0.59$ & $3.05 \pm 0.10$ & $51.10 \pm 3.19$ & $5.46 \pm 0.71 \mathrm{~b}$ & $1.73 \pm 0.12 b$ & $4.55 \pm 1.17 \mathrm{~b}$ & $25.35 \pm 2.92$ \\
\hline \multicolumn{8}{|l|}{ Salinity (S) } \\
\hline Control & $44.38 \pm 0.97$ & $3.36 \pm 0.10 \mathrm{a}$ & $48.70 \pm 2.40 \mathrm{~b}$ & $6.23 \pm 0.65 b$ & $2.24 \pm 0.21 \mathrm{a}$ & $2.01 \pm 0.24 b$ & $11.58 \pm 0.61 c$ \\
\hline $\mathrm{CaCl} 2$ & $43.85 \pm 0.76$ & $2.79 \pm 0.14 b$ & $46.33 \pm 3.18 b$ & $10.84 \pm 1.27 \mathrm{a}$ & $2.13 \pm 0.14 \mathrm{ab}$ & $2.05 \pm 0.23 b$ & $25.33 \pm 3.34 \mathrm{~b}$ \\
\hline $\mathrm{KCl}$ & $44.43 \pm 0.70$ & $3.19 \pm 0.08 \mathrm{a}$ & $68.05 \pm 4.14 \mathrm{a}$ & $3.93 \pm 0.28 c$ & $1.54 \pm 0.12 c$ & $1.73 \pm 0.20 \mathrm{~b}$ & $33.50 \pm 2.75 a$ \\
\hline $\mathrm{NaCl}$ & $45.04 \pm 0.60$ & $3.03 \pm 0.16 \mathrm{ab}$ & $40.14 \pm 2.73 b$ & $4.64 \pm 0.41 c$ & $1.73 \pm 0.15 b c$ & $14.63 \pm 1.4 \mathrm{a}$ & $27.21 \pm 5.26 \mathrm{~b}$ \\
\hline \multicolumn{8}{|l|}{ Cut (C) } \\
\hline Cut 1 & $46.04 \pm 0.41 \mathrm{a}$ & $2.85 \pm 0.08 b$ & $48.91 \pm 2.02$ & $4.91 \pm 0.40 \mathrm{~b}$ & $1.57 \pm 0.08 \mathrm{~b}$ & $4.60 \pm 0.87 \mathrm{a}$ & $15.38 \pm 1.35 b$ \\
\hline Cut 2 & $42.82 \pm 0.43 b$ & $3.33 \pm 0.08 \mathrm{a}$ & $52.70 \pm 3.84$ & $7.91 \pm 0.90 \mathrm{a}$ & $2.25 \pm 0.12 \mathrm{a}$ & $5.61 \pm 1.53 b$ & $33.44 \pm 2.80 \mathrm{a}$ \\
\hline \multicolumn{8}{|l|}{$\mathrm{CV} \times \mathrm{S}$} \\
\hline Control, Green & $45.41 \pm 0.90$ & $3.41 \pm 0.11$ & $48.69 \pm 1.97$ & $7.50 \pm 0.86$ & $2.55 \pm 0.27$ & $2.27 \pm 0.39$ & $11.42 \pm 0.69$ \\
\hline Control, Red & $43.35 \pm 1.70$ & $3.31 \pm 0.18$ & $48.70 \pm 4.63$ & $4.97 \pm 0.68$ & $1.93 \pm 0.29$ & $1.76 \pm 0.27$ & $11.75 \pm 1.09$ \\
\hline $\mathrm{CaCl} 2$,Green & $44.34 \pm 1.23$ & $2.94 \pm 0.23$ & $43.97 \pm 3.77$ & $12.18 \pm 1.64$ & $2.25 \pm 0.13$ & $2.24 \pm 0.42$ & $22.42 \pm 5.86$ \\
\hline $\mathrm{CaCl} 2, \mathrm{Red}$ & $43.37 \pm 0.96$ & $2.64 \pm 0.15$ & $48.70 \pm 5.30$ & $9.49 \pm 1.93$ & $2.01 \pm 0.26$ & $1.86 \pm 0.22$ & $28.25 \pm 3.38$ \\
\hline $\mathrm{KCl}$, Green & $44.41 \pm 1.05$ & $3.18 \pm 0.06$ & $70.38 \pm 4.24$ & $4.29 \pm 0.26$ & $1.63 \pm 0.12$ & $1.96 \pm 0.32$ & $32.67 \pm 3.31$ \\
\hline $\mathrm{KCl}$, Red & $44.45 \pm 1.02$ & $3.20 \pm 0.15$ & $65.73 \pm 7.42$ & $3.58 \pm 0.47$ & $1.44 \pm 0.20$ & $1.49 \pm 0.24$ & $34.33 \pm 4.70$ \\
\hline $\mathrm{NaCl}$, Green & $45.51 \pm 0.53$ & $3.01 \pm 0.26$ & $39.01 \pm 3.68$ & $5.49 \pm 0.43$ & $1.94 \pm 0.17$ & $16.17 \pm 1.51$ & $27.33 \pm 7.42$ \\
\hline \multicolumn{8}{|l|}{$\mathrm{S} \times \mathrm{C}$} \\
\hline Control, Cut 1 & $46.11 \pm 0.99$ & $3.10 \pm 0.10$ & $45.55 \pm 2.66$ & $4.62 \pm 0.46 \mathrm{~d}$ & $1.68 \pm 0.14$ & $2.59 \pm 0.30 c$ & $11.25 \pm 0.77 \mathrm{~d}$ \\
\hline Control, Cut 2 & $42.65 \pm 1.39$ & $3.62 \pm 0.09$ & $51.84 \pm 3.79$ & $7.85 \pm 0.77 \mathrm{~b}$ & $2.81 \pm 0.21$ & $1.43 \pm 0.16 c$ & $11.92 \pm 1.01 \mathrm{~d}$ \\
\hline $\mathrm{CaCl} 2$, Cut 1 & $45.68 \pm 0.73$ & $2.51 \pm 0.08$ & $47.95 \pm 3.34$ & $7.31 \pm 0.82 \mathrm{bc}$ & $1.83 \pm 0.15$ & $2.36 \pm 0.31 c$ & $15.25 \pm 2.62 \mathrm{~d}$ \\
\hline $\mathrm{CaCl} 2$, Cut 2 & $42.03 \pm 0.81$ & $3.06 \pm 0.22$ & $44.72 \pm 5.69$ & $14.36 \pm 1.22 \mathrm{a}$ & $2.43 \pm 0.18$ & $1.75 \pm 0.33 c$ & $35.42 \pm 1.29 \mathrm{~b}$ \\
\hline $\mathrm{KCl}$, Cut 1 & $46.06 \pm 0.87$ & $3.05 \pm 0.08$ & $59.32 \pm 4.43$ & $3.51 \pm 0.42 \mathrm{~d}$ & $1.27 \pm 0.11$ & $2.05 \pm 0.28 c$ & $24.67 \pm 0.46 c$ \\
\hline $\mathrm{KCl}$, Cut 2 & $42.80 \pm 0.57$ & $3.33 \pm 0.11$ & $76.79 \pm 5.01$ & $4.36 \pm 0.31 \mathrm{~d}$ & $1.80 \pm 0.13$ & $1.41 \pm 0.24 \mathrm{c}$ & $42.33 \pm 1.38 \mathrm{a}$ \\
\hline $\mathrm{NaCl}, \mathrm{Cut} 1$ & $46.29 \pm 0.88$ & $2.74 \pm 0.23$ & $42.82 \pm 2.42$ & $4.19 \pm 0.52 \mathrm{~d}$ & $1.50 \pm 0.14$ & $11.41 \pm 1.16 b$ & $10.33 \pm 0.57 \mathrm{~d}$ \\
\hline $\begin{array}{c}\mathrm{NaCl}, \text { Cut } 2 \\
\mathrm{CV} \times \mathrm{C}\end{array}$ & $43.79 \pm 0.42$ & $3.32 \pm 0.16$ & $37.46 \pm 4.91$ & $5.09 \pm 0.62 \mathrm{~cd}$ & $1.97 \pm 0.23$ & $17.85 \pm 1.77 \mathrm{a}$ & $44.08 \pm 2.71 \mathrm{a}$ \\
\hline Green, Cut 1 & $46.58 \pm 0.43$ & $2.96 \pm 0.13$ & $50.84 \pm 2.61$ & $6.03 \pm 0.53$ & $1.81 \pm 0.08$ & $5.58 \pm 1.44$ & $14.54 \pm 1.97$ \\
\hline Green, Cut 2 & $43.26 \pm 0.46$ & $3.31 \pm 0.13$ & $50.18 \pm 5.52$ & $8.69 \pm 1.37$ & $2.38 \pm 0.18$ & $5.74 \pm 2.29$ & $32.38 \pm 3.97$ \\
\hline Red, Cut 1 & $45.50 \pm 0.68$ & $2.73 \pm 0.09$ & $46.98 \pm 3.10$ & $3.79 \pm 0.41$ & $1.32 \pm 0.08$ & $3.62 \pm 0.96$ & $16.21 \pm 1.91$ \\
\hline Red, Cut 2 & $42.38 \pm 0.73$ & $3.36 \pm 0.11$ & $55.22 \pm 5.48$ & $7.13 \pm 1.19$ & $2.13 \pm 0.17$ & $5.48 \pm 2.15$ & $34.50 \pm 4.09$ \\
\hline \multicolumn{8}{|l|}{ Significance } \\
\hline Cultivar (CV) & n.s. & n.s. & n.s. & $* * *$ & $* *$ & $*$ & n.s. \\
\hline Salinity (S) & n.s. & $* *$ & $* * *$ & $* * *$ & $* * *$ & $* * *$ & $* * *$ \\
\hline Cut (C) & $* * *$ & $* * *$ & n.s. & $* * *$ & $* * *$ & $* * *$ & $* * *$ \\
\hline $\mathrm{CV} \times \mathrm{S}$ & n.s. & n.s. & n.s. & n.s. & n.s. & n.s. & n.s. \\
\hline $\mathrm{S} \times \mathrm{C}$ & n.s. & n.s. & n.s. & $* * *$ & n.s. & $* * *$ & $* * *$ \\
\hline $\mathrm{CV} \times \mathrm{C}$ & n.s. & n.s. & n.s. & n.s. & n.s. & n.s. & n.s. \\
\hline $\mathrm{CV} \times \mathrm{S} \times \mathrm{C}$ & n.s. & n.s. & n.s. & n.s. & n.s. & n.s. & n.s. \\
\hline
\end{tabular}

n.s., $* * * * * *$ Non-significant or significant at $p \leq 0.05,0.01$, and 0.001 , respectively. Different letters indicate statistically different groups $(p<0.05$, Duncan's post hoc test following ANOVA; $\mathrm{n}=3)$.

Overall, the $\mathrm{Na}$ content increased only with $\mathrm{NaCl}$ treatment, whereas the $\mathrm{Cl}$ content in leaf tissue increased in all chloride-based treatments $\left(\mathrm{CaCl}_{2}, \mathrm{KCl}\right.$, and $\left.\mathrm{NaCl}\right)$ in comparison to the non-saline control (Table 3). Furthermore, under the different salinity treatments, the accumulation behavior of the two cultivars for both ions was similar since the $\mathrm{CV} \times \mathrm{S}$ interaction was not significant. However, the sodium concentration in leaf tissue was 
significantly higher in green than in red baby lettuce. The $\mathrm{S} \times \mathrm{C}$ interaction was observed for both toxic ions as the $\mathrm{Na}$ content increased only under the $\mathrm{NaCl}$ treatment during the second cut while the control's $\mathrm{Cl}$ content was the only one remained unchanged among the salinity treatments during the second cut. Independently of the cultivars, the highest concentration of $\mathrm{Na}$ was recorded in the $\mathrm{NaCl}$ treatment, cut 2, and the highest $\mathrm{Cl}$ concentration in leaf tissue was attained in both $\mathrm{KCl}$ and $\mathrm{NaCl}$ treatments, cut 2 (Table 3).

\subsection{Anti-Nutrients, Antioxidant Activities, and Phytochemical Quantification}

Nitrate concentration was affected by salinity and cut treatments (Table 4). The cultivar $\times$ salinity interaction was significant since $\mathrm{KCl}$ and $\mathrm{CaCl}_{2}$ treatments decreased the nitrate content only in green lettuce when compared to the control. The salinity $\times$ cut interaction was also significant since the lettuce nitrate content was reduced during the second cut only when lettuce plants were treated with $\mathrm{CaCl}_{2}$ or $\mathrm{NaCl}$. The lowest nitrate values were recorded in lettuce plants fertigated with $13.3 \mathrm{mM} \mathrm{CaCl}_{2}$ harvested $45 \mathrm{DAS}$ (cut 2; Table 4).

Table 4. Significance of the main factors (cultivar, salinity, and cut order) and their interactions on nitrate content, lipophilic antioxidant activity [LAA], hydrophilic antioxidant activity [HAA], and total ascorbic acid [TAA] of Lactuca sativa L. var. acephala grown in a floating system.

\begin{tabular}{|c|c|c|c|c|}
\hline Source of Variance & $\begin{array}{c}\text { Nitrate } \\
\left(\mathrm{mg} \mathrm{kg}^{-1} \mathrm{fw}\right)\end{array}$ & $\begin{array}{c}\text { LAA } \\
\left(\mathrm{mmol} \text { Trolox } 100 \mathrm{~g}^{-1} \mathrm{dw}\right)\end{array}$ & $\begin{array}{c}\text { HAA } \\
\text { (mmol Ascorbic ac. eq. } \\
\left.\mathrm{kg}^{-1} \mathrm{dw}\right)\end{array}$ & $\begin{array}{c}\text { TAA } \\
\left(\mathrm{mg} 100 \mathrm{~g}^{-1} \mathrm{fw}\right)\end{array}$ \\
\hline \multicolumn{5}{|l|}{ Cultivar (CV) } \\
\hline Green & $1961.45 \pm 104.81$ & $3.72 \pm 0.15 b$ & $1.40 \pm 0.08$ & $46.81 \pm 3.00 \mathrm{~b}$ \\
\hline Red & $1917.22 \pm 93.35$ & $4.68 \pm 0.25 \mathrm{a}$ & $1.31 \pm 0.09$ & $67.23 \pm 4.09 \mathrm{a}$ \\
\hline \multicolumn{5}{|l|}{ Salinity (S) } \\
\hline Control & $2210.08 \pm 118.69 \mathrm{a}$ & $3.63 \pm 0.27 b$ & $1.27 \pm 0.03$ & $45.29 \pm 3.67 \mathrm{~b}$ \\
\hline $\mathrm{CaCl} 2$ & $1593.01 \pm 109.51 \mathrm{~b}$ & $4.58 \pm 0.3 \mathrm{a}$ & $1.43 \pm 0.06$ & $74.41 \pm 4.19 \mathrm{a}$ \\
\hline $\mathrm{KCl}$ & $1975.78 \pm 118.05 \mathrm{a}$ & $3.94 \pm 0.31 b$ & $1.38 \pm 0.04$ & $58.24 \pm 6.78 \mathrm{~b}$ \\
\hline $\mathrm{NaCl}$ & $1978.47 \pm 157.22 \mathrm{a}$ & $4.64 \pm 0.34 \mathrm{a}$ & $1.31 \pm 0.04$ & $50.14 \pm 4.90 \mathrm{~b}$ \\
\hline \multicolumn{5}{|l|}{ Cut (C) } \\
\hline Cut 1 & $2115.92 \pm 74.67 \mathrm{a}$ & $4.95 \pm 0.19 \mathrm{a}$ & $1.27 \pm 0.02 b$ & $54.83 \pm 4.87$ \\
\hline Cut 2 & $1762.75 \pm 107.03 \mathrm{~b}$ & $3.45 \pm 0.15 b$ & $1.42 \pm 0.03 \mathrm{a}$ & $59.21 \pm 3.26$ \\
\hline \multicolumn{5}{|l|}{$\mathrm{CV} \times \mathrm{S}$} \\
\hline Control, Green & $2366.26 \pm 167.12 \mathrm{a}$ & $3.22 \pm 0.27$ & $1.30 \pm 0.05$ & $35.55 \pm 2.51$ \\
\hline Control, Red & $2053.89 \pm 155.88 \mathrm{ab}$ & $4.04 \pm 0.42$ & $1.25 \pm 0.05$ & $55.02 \pm 3.90$ \\
\hline $\mathrm{CaCl} 2$,Green & $1530.81 \pm 137.77 \mathrm{c}$ & $4.02 \pm 0.21$ & $1.52 \pm 0.06$ & $63.97 \pm 2.31$ \\
\hline $\mathrm{CaCl} 2, \mathrm{Red}$ & $1655.21 \pm 179.56 \mathrm{bc}$ & $5.14 \pm 0.46$ & $1.33 \pm 0.10$ & $84.85 \pm 5.33$ \\
\hline $\mathrm{KCl}$, Green & $1754.21 \pm 108.87 \mathrm{bc}$ & $3.70 \pm 0.28$ & $1.40 \pm 0.08$ & $44.37 \pm 4.07$ \\
\hline $\mathrm{KCl}$, Red & $2197.36 \pm 172.71 \mathrm{ab}$ & $4.18 \pm 0.57$ & $1.36 \pm 0.02$ & $72.1 \pm 10.44$ \\
\hline $\mathrm{NaCl}$, Green & $2194.54 \pm 232.51 \mathrm{ab}$ & $3.94 \pm 0.34$ & $1.39 \pm 0.03$ & $43.33 \pm 7.02$ \\
\hline $\begin{array}{c}\mathrm{NaCl}, \mathrm{Red} \\
\mathrm{S} \times \mathrm{C}\end{array}$ & $1762.4 \pm 189.81 \mathrm{bc}$ & $5.35 \pm 0.43$ & $1.24 \pm 0.05$ & $56.96 \pm 6.14$ \\
\hline Control, Cut 1 & $2033.59 \pm 100.69 \mathrm{ab}$ & $4.35 \pm 0.29$ & $1.19 \pm 0.04 c$ & $41.38 \pm 3.06 \mathrm{~d}$ \\
\hline Control, Cut 2 & $2386.56 \pm 198.46 \mathrm{a}$ & $2.91 \pm 0.15$ & $1.35 \pm 0.03 \mathrm{bc}$ & $49.20 \pm 6.63 \mathrm{~cd}$ \\
\hline $\mathrm{CaCl} 2, \mathrm{Cut} 1$ & $1864.45 \pm 80.3 \mathrm{bc}$ & $5.21 \pm 0.45$ & $1.26 \pm 0.08 \mathrm{bc}$ & $71.58 \pm 6.06 \mathrm{ab}$ \\
\hline $\mathrm{CaCl} 2$, Cut 2 & $1321.57 \pm 129.81 \mathrm{~d}$ & $3.95 \pm 0.16$ & $1.59 \pm 0.04 \mathrm{a}$ & $77.24 \pm 6.13 a$ \\
\hline $\mathrm{KCl}$, Cut 1 & $2165.31 \pm 175.53 \mathrm{ab}$ & $4.76 \pm 0.27$ & $1.32 \pm 0.02 \mathrm{bc}$ & $64.86 \pm 13.17 \mathrm{abc}$ \\
\hline $\mathrm{KCl}, \mathrm{Cut} 2$ & $1786.25 \pm 127.04 \mathrm{bc}$ & $3.12 \pm 0.30$ & $1.44 \pm 0.07 \mathrm{ab}$ & $51.61 \pm 3.36 \mathrm{bcd}$ \\
\hline $\mathrm{NaCl}, \mathrm{Cut} 1$ & $2400.32 \pm 156.61 \mathrm{a}$ & $5.46 \pm 0.35$ & $1.32 \pm 0.03 \mathrm{bc}$ & $41.50 \pm 8.32 \mathrm{~d}$ \\
\hline $\begin{array}{c}\mathrm{NaCl}, \mathrm{Cut} 2 \\
\mathrm{CV} \times \mathrm{C}\end{array}$ & $1556.62 \pm 114.24 \mathrm{c}$ & $3.82 \pm 0.33$ & $1.31 \pm 0.07 \mathrm{bc}$ & $58.79 \pm 2.52 \mathrm{abcd}$ \\
\hline Green, Cut 1 & $2123.77 \pm 118.33$ & $4.21 \pm 0.13 b$ & $1.32 \pm 0.03$ & $41.91 \pm 4.27$ \\
\hline Green, Cut 2 & $1799.14 \pm 164.77$ & $3.22 \pm 0.16 c$ & $1.48 \pm 0.05$ & $51.70 \pm 3.87$ \\
\hline Red, Cut 1 & $2108.06 \pm 96.45$ & $5.68 \pm 0.17 \mathrm{a}$ & $1.23 \pm 0.04$ & $67.74 \pm 7.12$ \\
\hline Red, Cut 2 & $1726.37 \pm 143.24$ & $3.68 \pm 0.24 c$ & $1.36 \pm 0.04$ & $66.72 \pm 4.39$ \\
\hline
\end{tabular}


Table 4. Cont

\begin{tabular}{|c|c|c|c|c|}
\hline Source of Variance & $\begin{array}{c}\text { Nitrate } \\
\left(\mathrm{mg} \mathrm{kg}^{-1} \text { fw) }\right.\end{array}$ & $\begin{array}{c}\text { LAA } \\
\left(\mathrm{mmol} \text { Trolox } 100 \mathrm{~g}^{-1} \mathrm{dw}\right)\end{array}$ & $\begin{array}{c}\text { HAA } \\
\text { (mmol Ascorbic ac. eq. } \\
\mathrm{kg}^{-1} \mathrm{dw} \text { ) }\end{array}$ & $\begin{array}{c}\text { TAA } \\
\left(\mathrm{mg} 100 g^{-1} \mathrm{fw}\right)\end{array}$ \\
\hline \multicolumn{5}{|l|}{ Significance } \\
\hline Cultivar (CV) & n.s. & $* * *$ & n.s. & $* * *$ \\
\hline Salinity (S) & $* * *$ & $* * *$ & $* *$ & $* * *$ \\
\hline Cut (C) & $* * *$ & $* * *$ & $* * *$ & n.s. \\
\hline $\mathrm{CV} \times \mathrm{S}$ & $* * *$ & n.s. & n.s. & n.s. \\
\hline$S \times C$ & $* * *$ & n.s. & $* *$ & \\
\hline $\mathrm{CV} \times \mathrm{C}$ & n.s. & $* * *$ & n.s. & n.s. \\
\hline $\mathrm{CV} \times \mathrm{S} \times \mathrm{C}$ & n.s. & n.s. & n.s. & n.s. \\
\hline
\end{tabular}

n.s., ${ }^{*}, * * * * *$ Non-significant or significant at $p \leq 0.05,0.01$, and 0.001 , respectively. Different letters indicate statistically different groups $(p<0.05$, Duncan's post hoc test following ANOVA; $\mathrm{n}=3)$

Lipophilic antioxidant activity (LAA) was significantly affected by all the factors examined during this study (Table 4). A significant cultivar $\times$ cut interaction revealed that red type lettuce's LAA was higher than the green type only during the first cut. Nevertheless, both lettuces' LAA was reduced during the second cut, and the percentage of LAA reduction in leaf tissue caused by cut order was significantly higher $(35.2 \%)$ in the red lettuce cultivar compared to the one recorded in the green pigmented variety $(23.7 \%)$. The LAA was increased by all salinity treatments compared to the control, except the $\mathrm{KCl}$ treatment, which was not significantly different from the non-saline control. Hydrophilic antioxidant activity (HAA) of the two cultivars was similar but it was affected by salinity and the successive cuts. $\mathrm{CaCl}_{2}$ was the only salinity treatment which increased HAA compared to the control. A salinity $\times$ cut interaction was observed for HAA as no salinity treatment affected HAA during the first cut while during the second cut, $\mathrm{CaCl}_{2}$ yielded higher HAA than some of the other treatments.

TAA was also influenced by salinity treatments. A salinity $\times$ cut interaction was observed since TAA was increased, when compared to the control, by both $\mathrm{CaCl}_{2}$ and $\mathrm{KCl}$ during the first cut but only by $\mathrm{CaCl}_{2}$ during the second cut.

\subsection{Principal Component Analysis (PCA)}

A principal component analysis was performed on all lettuce-analyzed data in relation to the three tested experimental factors: cultivars, iso-osmotic salt concentrations, and successive harvests, and the loading plot and scores are reported in Figure 1. The variables in the first four principal components (PCs) were highly correlated, with eigen values greater than 1, thus explaining $81.0 \%$ of the total variance, with PC1, PC2, PC3, and PC4 accounting for $34.0 \%, 20.9 \%, 13.8 \%$, and $12.2 \%$, respectively. PC1 was positively correlated with dry biomass, $\mathrm{Mg}$, leaf area, fresh yield, and leaf dry matter, while it was negatively correlated with LAA. Moreover, PC2 was positively correlated with a*, Cl, TAA, and TSS, while it was negatively correlated with $b^{*}, L^{*}$, and total $\mathrm{N}$. The two baby lettuce cultivars were well separated but not uniformly clustered with respect to PC1 and PC2. In fact, all three factors examined in this study were important in PCA clustering along PC2 and PC1. In particular, green lettuce treatments from cut 2 were distributed on the positive side of PC1 between upper and lower right quadrants, while red lettuce from the cut 1 treatment was distributed on the negative side of PC1, mostly in the upper left quadrant. Moreover, the green lettuce from the cut 1 treatment was distributed in the negative side of PC2 between the lower right and left quadrants, while red lettuce from the cut 2 treatment was on the positive side of PC2 in the upper right quadrant close to the $\mathrm{Y}$ axis (Figure 1). Interestingly, the combination green cultivar-cut 2 under control and $\mathrm{CaCl}_{2}$ conditions showed the highest dry biomass, $\mathrm{Mg}$, leaf area, fresh yield, and leaf dry matter, whereas the red cultivar produced a higher premium quality lettuce (TAA during both harvests and LAA during the first cut; Figure 1). 


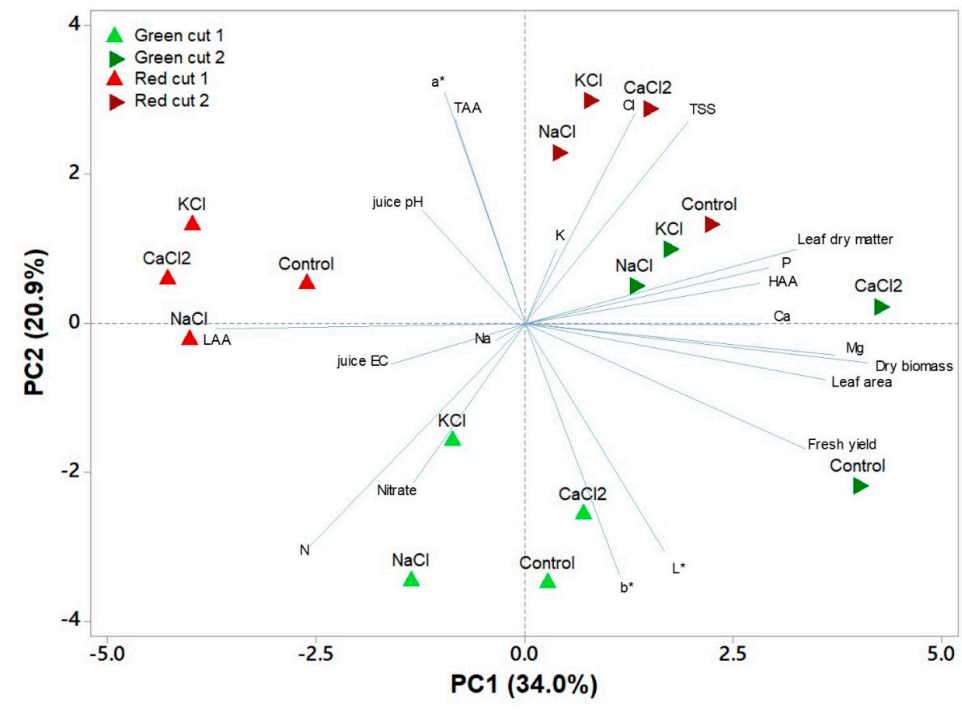

Figure 1. Principal component loading plot and scores of principal component analysis (PCA) of biometric traits, leaf colorimetry, flavor compounds, mineral composition, bioactive compounds, and antioxidant activity of green and red pigmented lettuces under four salinity treatments (control, $\mathrm{CaCl}_{2}, \mathrm{KCl}$, and $\mathrm{NaCl}$ ) and two successive harvests.

\section{Discussion}

In our study, we applied mild iso-osmotic concentrations of three different salts combined with two successive harvests to green and red pigmented lettuce to define the best possible treatment for enhancing the nutritional quality of produce without exerting a negative impact on its growth and yield.

Green lettuce had a significant higher productivity concerning leaf area, fresh yield, and dry biomass compared to red lettuce, as also found in previous studies on the same cultivars [8,49]. In particular, Carillo, et al. [8] showed that this feature could depend on a higher intrinsic water use efficiency $\left(\mathrm{WUE}_{\mathrm{i}}\right)$ and stomatal conductance, which implied a greater availability of $\mathrm{CO}_{2}$ and a faster and more efficient photosynthetic electron transport chain, which may lead to a lower production of harmful reactive oxygen species (ROS) [50]. Indeed, this resulted in a lower demand for antioxidant metabolites, as proven by the significantly lower amounts of TAA in the green cultivar compared to the red one. Since the cost in terms of energy for the synthesis of organic compounds for oxidative stress or osmotic protection may be very high (50-70 moles ATP for mole) [51,52], the high energy savings could be used for plant growth. The more efficient light-dependent reactions of photosynthesis could also be a consequence of the higher efficiency of the reductive pentose phosphate pathway (Benson-Calvin cycle) in the presence of higher concentrations of $\mathrm{Mg}$, as found in the green cultivar [53]. Besides, $\mathrm{Mg}$ is not only essential for the activation of Rubisco, which requires the reversible binding of $\mathrm{CO}_{2}$ and $\mathrm{Mg}$ to its active site, but also for the light-dependent increase in activity of Calvin cycle enzymes fructose 1,6-bisphosphatase and sedoheptulose 1,7-bisphosphatase, which is accompanied by an alkalization and an increase in the $\mathrm{Mg}$ concentration [54].

At these iso-osmotic concentrations, only $\mathrm{KCl}$ and $\mathrm{NaCl}$ were able to significantly, decrease marketable yield, while there were no differences between the control and $\mathrm{CaCl}_{2}$ treatments. $\mathrm{Na}$ and $\mathrm{Cl}$ can be toxic when their concentration rises in the cytosol and organelles [55]. It is interesting to note that $\mathrm{Na}$ strongly accumulated in NaCl-treated plants at cut 1 and further increased at cut 2. $\mathrm{Cl}$ increased at cut 1 only under $\mathrm{KCl}$ treatment, while it strongly increased under all salt treatments at cut 2. However, in the same plants, $\mathrm{K}$ remained stable and this could account for the slight effect on yield since the cytosolic toxicity of $\mathrm{Na}$ and $\mathrm{Cl}$ is not only dependent on their absolute amount but is related to the $\mathrm{K}$ to $\mathrm{Na}$ ratio and the ability of cells to compartmentalize the toxic ions in the vacuole $[51,56,57]$. In fact, $K$, even at low concentrations, when compartmentalized 
in the cytosol, which occupies less than $10 \%$ of the cell volume, can generate a notable osmotic pressure able to oppose vacuolar osmotic potential, reducing salt stress effects and restoring the ability of plant tissues to grow [51,58].

Indeed, the tolerance of lettuce plants to the iso-osmotic concentrations of salts may depend on the ability of plants to retain $\mathrm{Mg}$ and $\mathrm{K}$ while sequestering toxic ions in the vacuole. However, equally important is the capacity to fine-tune metabolic pathways in order to synthetize protecting metabolites able to revert stress damages. [21,59-61]. Besides, $\mathrm{CaCl}_{2}$ and $\mathrm{NaCl}$ treatments increased LAA, probably because the lipophilic antioxidant content, related to carotenoids, chlorophylls, and tocopherols [62], was potentiated under salinity to protect the photosynthetic apparatus, positively impacting nutritional quality and the shelf life of the produce. However, based on the Pearson correlation, LAA was negatively correlated with leaf area in red lettuce $(r=0.80)$ and was lower at cut 2 . This means that while at cut 1 red lettuce invested more energy in preserving the photosynthetic apparatus, at cut 2, the main effort (energy) was spent on tissue expansion as also found by Corrado, et al. [63]. In fact, the total leaf area and thus fresh yield were significantly lower in red lettuce cut 1 compared to other treatments $(\mathrm{CV} \times \mathrm{C}$ interaction), while its antioxidant response was highly increased. The slight decrease in the yield of red lettuce compared to green lettuce at cut 1 was largely compensated by the higher quality of produce. In fact, the significant increase in TAA could not only favor a longer post-harvest life of produce, but also, since the most phenolics are flavonoids, a more intense color of red lettuce, as proven by decrease in leaf brightness $\left(L^{*}\right)$ and leaf green intensity (i.e., negative $a^{*}$ values) [18]. According to Neocleous, Koukounaras, Siomos, and Vasilakakis [31], darker green leaves could be a symptom of higher osmotic pressure and reduced water uptake; however, at cut 1 , red lettuce did not show higher TSS but only a higher juice EC than the green cultivar. The changes in the visual appearance of red lettuce at cut 1 could positively influence consumers' preferences and purchasing behavior, since color has also been identified as a reliable marker of the antioxidant properties of vegetables [9,17,25,27,64]. Indeed, ascorbic acid plays a pivotal role in the antioxidant response by scavenging free radicals and quenching singlet oxygen [29].

The increase in leaf area and fresh yield of the red cultivar at the second cut was much greater so as to match the values of the green one. This was probably due to an increase in the TSS content due to an enhanced accumulation of sugars in plant tissues, with less used for synthesizing secondary metabolites, and able to decrease the water potential and drive water influx responsible for tissue expansion [40].

In this study, the nitrate content decreased during the second harvest, in contrast to Corrado, et al. [63] and Carillo, et al. [8], who recorded higher contents of nitrate in basil leaves and red and green lettuce, respectively, during the last harvest. However, the nitrate content, independently of harvest and salt treatments, remained within the limits established by EU regulation no. 1258/2011 (e.g., lower than 3000-5000 mg kg-1 fw). In control plants, nitrate reduction at cut 2 was probably due to the fact that lettuce plants grown in hydroponics were supplied with an equal and constant amount of nitrogen fertilization until the end of cultivation while the plant dimensions (leaf area and probably also leaf number and root volume) increased with the use and/or dilution of the absorbed nitrate. Moreover, from cut 1 to cut 2, a decrease in the nitrate concentration was observed. This could be linked mainly to environmental factors. These include the increase in light intensity (data not shown) that certainly played an important role in reducing the nitrate content by promoting the activity of nitrate reductase during May compared to April. Whereas in salt treated plants in which the concentration of $\mathrm{Cl}$ was much higher at cut 2 , independently of the type of salt applied, there was certainly an inhibition of uptake and xylem loading of nitrate caused by the competition between $\mathrm{NO}_{3}$ and $\mathrm{Cl}$ [65]. In fact, $\mathrm{Cl}$ is able to specifically interfere with $\mathrm{NO}_{3}$ root uptake, transport, and xylem loading by using the same anion channels used by $\mathrm{NO}_{3}[65,66]$. However, the significantly higher leaf content of $\mathrm{Cl}$, as well as of $\mathrm{Ca}, \mathrm{P}, \mathrm{Mg}$, and $\mathrm{Na}$, at cut 2 may also depend on the higher 
leaf area, driving a higher transpiration stream in the presence of water availability able to increase ion xylem transport [67].

\section{Conclusions}

A mild to moderate nutritional stress (eustress) can remodulate plant metabolism by inducing the synthesis and accumulation of beneficial metabolites, improving the plant defense system, productivity, and quality of the final products. However, it is necessary to expertly administer the right dose of stress/eustress in order to avoid that the activation of the defence system, and the synthesis of these new protective metabolites could affect plant growth and yield. Our study showed that green lettuce had superior biometric traits and productivity response compared to red lettuce at the first harvest but also a lower nutraceutical quality and post-harvest duration because of the lower TAA content compared to red lettuce. However, at the iso-osmotic concentration of salts used, neither of the two cultivars showed a higher sensitivity to salinity probably due to their high capacity to retain $\mathrm{K}$ and $\mathrm{Mg}$. Moreover, a positive effect of the mild iso-osmotic salinity treatments for both cultivars at cut 1 was the increase in the lipophilic antioxidant content (LAA). The cut order was also important, independently of salinity treatments, which showed that while at the second harvest no significant effects were found concerning phytonutrients, the antinutrient nitrate content decreased while fresh weight increased in the red cultivar.

Author Contributions: Conceptualization, Y.R.; methodology, Y.R. and P.C.; software, M.G. and G.A.S.; validation, M.G. and G.A.S.; formal analysis, I.D.M., M.M.; investigation, M.G., G.R., F.N. and E.D.S.; resources, Y.R.; data curation, F.N., M.G., G.R. and E.D.S.; writing-original draft preparation, P.C. and Y.R.; writing-review and editing, P.C., G.A.S., M.C.K., M.G., G.R., F.N., E.D.S., I.D.M., M.M. and Y.R.; visualization, P.C. and Y.R.; supervision, P.C. and Y.R.; project administration, Y.R.; funding acquisition, P.C. and Y.R. All authors have read and agreed to the published version of the manuscript.

Funding: This research received no external funding.

Institutional Review Board Statement: Not applicable.

Informed Consent Statement: Not applicable.

Data Availability Statement: The datasets generated for this study are available on request to the corresponding author.

Acknowledgments: The authors are grateful to Arcangelo Ambrosio for his assistance in the greenhouse experiment.

Conflicts of Interest: The authors declare no conflict of interest.

\section{References}

1. MarketQuest.Biz. Global Leafy Greens Seeds Market 2020 by Manufacturers, Regions, Type and Application, Forecast to 2025. Report ID: 35273. Available online: https:/ / www.marketquest.biz/report/35273/global-leafy-greens-seeds-market-2020-bymanufacturers-regions-type-and-application-forecast-to-2025 (accessed on 1 March 2021).

2. Pignata, G.; Ertani, A.; Casale, M.; Piano, P.; Nicola, S. Mixing fresh-cut baby green and red leaf lettuce from soilless cultivation preserves phytochemical content and safety. Agric. Food Sci. 2020, 29. [CrossRef]

3. Ciriello, M.; Formisano, L.; El-Nakhel, C.; Kyriacou, M.C.; Soteriou, G.A.; Pizzolongo, F.; Romano, R.; De Pascale, S.; Rouphael, Y. Genotype and successive harvests interaction affects phenolic acids and aroma profile of Genovese basil for pesto sauce production. Foods 2021, 10, 278. [CrossRef] [PubMed]

4. Carillo, P.; Morrone, B.; Fusco, G.M.; De Pascale, S.; Rouphael, Y. Challenges for a sustainable food production system on board of the international space station: A technical review. Agronomy 2020, 10, 687. [CrossRef]

5. Pepe, G.; Sommella, E.; Manfra, M.; De Nisco, M.; Tenore, G.C.; Scopa, A.; Sofo, A.; Marzocco, S.; Adesso, S.; Novellino, T.; et al. Evaluation of anti-inflammatory activity and fast UHPLC-DAD-IT-TOF profiling of polyphenolic compounds extracted from green lettuce (Lactuca sativa L.; var. Maravilla de Verano). Food Chem. 2015, 167, 153-161. [CrossRef]

6. Arienzo, A.; Murgia, L.; Fraudentali, I.; Gallo, V.; Angelini, R.; Antonini, G. Microbiological quality of ready-to-eat leafy green salads during shelf-life and home-refrigeration. Foods 2020, 9, 1421. [CrossRef] 
7. Carillo, P.; Colla, G.; Fusco, G.M.; Dell'Aversana, E.; El-Nakhel, C.; Giordano, M.; Pannico, A.; Cozzolino, E.; Mori, M.; Reynaud, H.; et al. Morphological and physiological responses induced by protein hydrolysate-based biostimulant and nitrogen rates in greenhouse spinach. Agronomy 2019, 9, 450. [CrossRef]

8. Carillo, P.; Giordano, M.; Raimondi, G.; Napolitano, F.; Di Stasio, E.; Kyriacou, M.C.; Sifola, M.I.; Rouphael, Y. Physiological and nutraceutical quality of green and red pigmented lettuce in response to nacl concentration in two successive harvests. Agronomy 2020, 10, 1358. [CrossRef]

9. Alvino, A.; Barbieri, G. Vegetables of temperate climates: Leafy vegetables. In The Encyclopedia of Food and Health; Oxford University Press: Oxford, UK, 2016; pp. 393-400. [CrossRef]

10. Llorach, R.; Martínez-Sánchez, A.; Tomás-Barberán, F.; Gil, M.; Ferreres, F. Characterisation of polyphenols and antioxidant properties of five lettuce varieties and escarole. Food Chem. 2008, 108, 1028-1038. [CrossRef]

11. Mulabagal, V.; Ngouajio, M.; Nair, A.; Zhang, Y.; Gottumukkala, A.; Nair, M. In vitro evaluation of red and green lettuce (Lactuca sativa) for functional food properties. Food Chem. 2010, 118, 300-306. [CrossRef]

12. Durazzo, A.; Azzini, E.; Lazzé, M.; Raguzzini, A.; Pizzala, R.; Maiani, G.; Palomba, L. Antioxidants in Italian head lettuce (Lactuca sativa var. capitatal.) grown in organic and conventional systems under greenhouse conditions. J. Food Biochem. 2013, 38. [CrossRef]

13. Ribas-Agustí, A.; Gratacós-Cubarsí, M.; Sárraga, C.; García-Regueiro, J.-A.; Castellari, M. Analysis of eleven phenolic compounds including novel p-coumaroyl derivatives in lettuce (Lactuca sativa L.) by ultra-high-performance liquid chromatography with photodiode array and mass spectrometry detection. Phytochem. Anal. 2011, 22, 555-563. [CrossRef] [PubMed]

14. Sofo, A.; Lundegårdh, B.; Mårtensson, A.; Manfra, M.; Pepe, G.; Sommella, E.; De Nisco, M.; Tenore, G.C.; Campiglia, P.; Scopa, A. Different agronomic and fertilization systems affect polyphenolic profile, antioxidant capacity and mineral composition of lettuce. Sci. Hortic. 2016, 204, 106-115. [CrossRef]

15. Shahidi, F.; Ambigaipalan, P. Phenolics and polyphenolics in foods, beverages and spices: Antioxidant activity and health effects-A review. J. Funct. Foods 2015, 18, 820-897. [CrossRef]

16. Złotek, U.; Świeca, M.; Jakubczyk, A. Effect of abiotic elicitation on main health-promoting compounds, antioxidant activity and commercial quality of butter lettuce (Lactuca sativa L.). Food Chem. 2014, 148, 253-260. [CrossRef] [PubMed]

17. Kim, M.J.; Moon, Y.; Tou, J.C.; Mou, B.; Waterland, N.L. Nutritional value, bioactive compounds and health benefits of lettuce (Lactuca sativa L.). J. Food Compos. Anal. 2016, 49, 19-34. [CrossRef]

18. Kim, H.-J.; Fonseca, J.M.; Choi, J.-H.; Kubota, C.; Kwon, D.Y. Salt in Irrigation Water Affects the Nutritional and Visual Properties of Romaine Lettuce (Lactuca sativa L.). J. Agric. Food Chem. 2008, 56, 3772-3776. [CrossRef]

19. Rouphael, Y.; Carillo, P.; Colla, G.; Fiorentino, N.; Sabatino, L.; El-Nakhel, C.; Giordano, M.; Pannico, A.; Cirillo, V.; Shabani, E.; et al. Appraisal of combined applications of trichoderma virens and a biopolymer-based biostimulant on lettuce agronomical, physiological, and qualitative properties under variable n regimes. Agronomy 2020, 10, 196. [CrossRef]

20. Rouphael, Y.; Vitaglione, P.; Colla, G.; Napolitano, F.; Raimondi, G.; Kyriacou, M.; Colantuono, A.; Giordano, M.; Pannico, A.; Maiello, R.; et al. Influence of mild saline stress and growing season on yield and leaf quality of baby lettuce grown in floating system. Acta Hortic. 2019, 1242, 147-152. [CrossRef]

21. Rouphael, Y.; Kyriacou, M.C. Enhancing Quality of Fresh Vegetables through Salinity Eustress and Biofortification Applications Facilitated by Soilless Cultivation. Front. Plant Sci. 2018, 9. [CrossRef]

22. Akula, R.; Ravishankar, G.A. Influence of abiotic stress signals on secondary metabolites in plants. Plant Signal. Behav. 2011, 6, 1720-1731. [CrossRef]

23. Woodrow, P.; Ciarmiello, L.F.; Annunziata, M.G.; Pacifico, S.; Iannuzzi, F.; Mirto, A.; D'Amelia, L.; Dell'Aversana, E.; Piccolella, S.; Fuggi, A.; et al. Durum wheat seedling responses to simultaneous high light and salinity involve a fine reconfiguration of amino acids and carbohydrate metabolism. Physiol. Plant. 2017, 159, 290-312. [CrossRef]

24. Lucini, L.; Borgognone, D.; Rouphael, Y.; Cardarelli, M.; Bernardi, J.; Colla, G. Mild potassium chloride stress alters the mineral composition, hormone network, and phenolic profile in artichoke leaves. Front. Plant Sci. 2016, 7, 948. [CrossRef] [PubMed]

25. Kyriacou, M.C.; Rouphael, Y. Towards a new definition of quality for fresh fruits and vegetables. Sci. Hortic. 2018, 234, 463-469. [CrossRef]

26. Giordano, M.; El-Nakhel, C.; Pannico, A.; Kyriacou, M.C.; Stazi, S.R.; De Pascale, S.; Rouphael, Y. Iron biofortification of red and green pigmented lettuce in closed soilless cultivation impacts crop performance and modulates mineral and bioactive composition. Agronomy 2019, 9, 290. [CrossRef]

27. Rouphael, Y.; Kyriacou, M.C.; Carillo, P.; Pizzolongo, F.; Romano, R.; Sifola, M.I. Chemical eustress elicits tailored responses and enhances the functional quality of novel food perilla frutescens. Molecules 2019, 24, 185. [CrossRef] [PubMed]

28. Neocleous, D.; Koukounaras, A.; Siomos, A.S.; Vasilakakis, M. Assessing the salinity effects on mineral composition and nutritional quality of green and red "baby" lettuce. J. Food Qual. 2014, 37, 1-8. [CrossRef]

29. Borgognone, D.; Cardarelli, M.; Rea, E.; Lucini, L.; Colla, G. Salinity source-induced changes in yield, mineral composition, phenolic acids and flavonoids in leaves of artichoke and cardoon grown in floating system. J. Sci. Food Agric. 2014, 94, $1231-1237$. [CrossRef] [PubMed]

30. Petropoulos, S.A.; Levizou, E.; Ntatsi, G.; Fernandes, Â.; Petrotos, K.; Akoumianakis, K.; Barros, L.; Ferreira, I.C.F.R. Salinity effect on nutritional value, chemical composition and bioactive compounds content of Cichorium spinosum L. Food Chem. 2017, 214, 129-136. [CrossRef] 
31. Neocleous, D.; Koukounaras, A.; Siomos, A.; Vasilakakis, M. Changes in Photosynthesis, Yield, and Quality of Baby Lettuce under Salinity Stress. J. Agric. Sci. Technol. 2014, 16, 1335-1343.

32. Scuderi, D.; Restuccia, C.; Chisari, M.; Barbagallo, R.N.; Caggia, C.; Giuffrida, F. Salinity of nutrient solution influences the shelf-life of fresh-cut lettuce grown in floating system. Postharvest Biol. Technol. 2011, 59, 132-137. [CrossRef]

33. Shin, Y.K.; Bhandari, S.R.; Jo, J.S.; Song, J.W.; Cho, M.C.; Yang, E.Y.; Lee, J.G. Response to salt stress in lettuce: Changes in chlorophyll fluorescence parameters, phytochemical contents, and antioxidant activities. Agronomy 2020, 10, 1627. [CrossRef]

34. Adhikari, N.D.; Simko, I.; Mou, B. Phenomic and physiological analysis of salinity effects on lettuce. Sensors 2019, 19 , 4814. [CrossRef] [PubMed]

35. Mahmoudi, H.; Huang, J.; Gruber, M.Y.; Kaddour, R.; Lachaâl, M.; Ouerghi, Z.; Hannoufa, A. The impact of genotype and salinity on physiological function, secondary metabolite accumulation, and antioxidative responses in lettuce. J. Agric. Food Chem. 2010, 58, 5122-5130. [CrossRef] [PubMed]

36. Ntatsi, G.; Aliferis, K.A.; Rouphael, Y.; Napolitano, F.; Makris, K.; Kalala, G.; Katopodis, G.; Savvas, D. Salinity source alters mineral composition and metabolism of Cichorium spinosum. Environ. Exp. Bot. 2017, 141, 113-123. [CrossRef]

37. Cirillo, C.; De Micco, V.; Arena, C.; Carillo, P.; Pannico, A.; De Pascale, S.; Rouphael, Y. Biochemical, Physiological and Anatomical Mechanisms of Adaptation of Callistemon citrinus and Viburnum lucidum to $\mathrm{NaCl}$ and $\mathrm{CaCl}(2)$ Salinization. Front. Plant Sci. 2019, 10, 742. [CrossRef]

38. Scagel, C.F.; Bryla, D.R.; Lee, J. Salt exclusion and mycorrhizal symbiosis increase tolerance to $\mathrm{NaCl}_{\text {and }} \mathrm{CaCl}_{2} \mathrm{Salinity}$ in 'Siam Queen' Basil. HortScience 2017, 52, 278-287. [CrossRef]

39. Borghesi, E.; Carmassi, G.; Uguccioni, M.C.; Vernieri, P.; Malorgio, F. Effects of calcium and salinity stress on quality of lettuce in soilless culture. J. Plant Nutr. 2013, 36, 677-690. [CrossRef]

40. Colla, G.; Rouphael, Y.; Jawad, R.; Kumar, P.; Rea, E.; Cardarelli, M. The effectiveness of grafting to improve $\mathrm{NaCl}_{\text {and }} \mathrm{CaCl} 2$ tolerance in cucumber. Sci. Hortic. 2013, 164, 380-391. [CrossRef]

41. Nicoletto, C.; Santagata, S.; Bona, S.; Sambo, P. Influence of cut number on qualitative traits in different cultivars of sweet basil. Ind. Crop. Prod. 2013, 44, 465-472. [CrossRef]

42. Petropoulos, S.; Fernandes, Â.; Karkanis, A.; Ntatsi, G.; Barros, L.; Ferreira, I.C.F.R. Successive harvesting affects yield, chemical composition and antioxidant activity of Cichorium spinosum L. Food Chem. 2017, 237, 83-90. [CrossRef]

43. Corrado, G.; Formisano, L.; De Micco, V.; Pannico, A.; Giordano, M.; El-Nakhel, C.; Chiaiese, P.; Sacchi, R.; Rouphael, Y. Understanding the morpho-anatomical, physiological, and functional response of sweet basil to isosmotic nitrate to chloride ratios. Biology 2020, 9, 158. [CrossRef] [PubMed]

44. Bremner, J.M. Total Nitrogen. In Methods of Soil Analysis; Black, C.A., Ed.; American Society of Agronomy: Madison, WN, USA, 1965; Volume 2, pp. 1149-1178.

45. Rouphael, Y.; Colla, G.; Graziani, G.; Ritieni, A.; Cardarelli, M.; De Pascale, S. Phenolic composition, antioxidant activity and mineral profile in two seed-propagated artichoke cultivars as affected by microbial inoculants and planting time. Food Chem. 2017, 234. [CrossRef] [PubMed]

46. Kampfenkel, K.; Van Montagu, M.; Inzé, D. Extraction and determination of ascorbate and dehydroascorbate from plant tissue. Anal. Biochem. 1995, 225, 165-167. [CrossRef] [PubMed]

47. Re, R.; Pellegrini, N.; Proteggente, A.; Pannala, A.; Yang, M.; Rice-Evans, C. Antioxidant activity applying an improved ABTS radical cation decolorization assay. Free Radic. Biol. Med. 1999, 26, 1231-1237. [CrossRef]

48. Fogliano, V.; Verde, V.; Randazzo, G.; Ritieni, A. Method for measuring antioxidant activity and its application to monitoring the antioxidant capacity of wines. J. Agric. Food Chem. 1999, 47, 1035-1040. [CrossRef] [PubMed]

49. El-Nakhel, C.; Giordano, M.; Pannico, A.; Carillo, P.; Fusco, G.M.; De Pascale, S.; Rouphael, Y. Cultivar-specific performance and qualitative descriptors for butterhead salanova lettuce produced in closed soilless cultivation as a candidate salad crop for human life support in space. Life 2019, 9, 61. [CrossRef] [PubMed]

50. Poljsak, B. Strategies for reducing or preventing the generation of oxidative stress. Oxidative Med. Cell. Longev. 2011, $2011,194586$. [CrossRef]

51. Annunziata, M.G.; Ciarmiello, L.F.; Woodrow, P.; Maximova, E.; Fuggi, A.; Carillo, P. Durum wheat roots adapt to salinity remodeling the cellular content of nitrogen metabolites and sucrose. Front. Plant Sci. 2017, 7. [CrossRef] [PubMed]

52. Raven, J.A. Tansley Review No. 2. Regulation of $\mathrm{pH}$ and generation of osmolarity in vascular plants: A cost-benefit analysis in relation to efficiency of use of energy, nitrogen and water. New Phytol. 1985, 101, 25-77. [CrossRef]

53. Verbruggen, N.; Hermans, C. Physiological and molecular responses to magnesium nutritional imbalance in plants. Plant Soil 2013, 368. [CrossRef]

54. Heldt, H.-W.; Heldt, F. 6-The Calvin cycle catalyzes photosynthetic CO2 assimilation. In Plant Biochemistry, 3rd ed.; Heldt, H.-W., Heldt, F., Eds.; Academic Press: Burlington, NJ, USA, 2005; pp. 165-193. [CrossRef]

55. Carillo, P.; Cirillo, C.; De Micco, V.; Arena, C.; De Pascale, S.; Rouphael, Y. Morpho-anatomical, physiological and biochemical adaptive responses to saline water of Bougainvillea spectabilis Willd. trained to different canopy shapes. Agric. Water Manag. 2019, 212, 12-22. [CrossRef]

56. Shabala, S.; Cuin, T.A. Potassium transport and plant salt tolerance. Physiol. Plant. 2008, 133, 651-669. [CrossRef] [PubMed] 
57. Rouphael, Y.; Raimondi, G.; Lucini, L.; Carillo, P.; Kyriacou, M.C.; Colla, G.; Cirillo, V.; Pannico, A.; El-Nakhel, C.; De Pascale, S. Physiological and metabolic responses triggered by omeprazole improve tomato plant Tolerance to NaCl Stress. Front. Plant Sci. 2018, 9, 249. [CrossRef] [PubMed]

58. Cuin, T.A.; Tian, Y.; Betts, S.A.; Chalmandrier, R.; Shabala, S. Ionic relations and osmotic adjustment in durum and bread wheat under saline conditions. Funct. Plant Biol. 2009, 36, 1110-1119. [CrossRef] [PubMed]

59. Rouphael, Y.; Kyriacou, M.C.; Petropoulos, S.A.; De Pascale, S.; Colla, G. Improving vegetable quality in controlled environments. Sci. Hortic. 2018, 234, 275-289. [CrossRef]

60. Ferchichi, S.; Hessini, K.; Dell'Aversana, E.; D’Amelia, L.; Woodrow, P.; Ciarmiello, L.F.; Fuggi, A.; Carillo, P. Hordeum vulgare and Hordeum maritimum respond to extended salinity stress displaying different temporal accumulation pattern of metabolites. Funct. Plant Biol. 2018, 45, 1096-1109. [CrossRef] [PubMed]

61. Song, Y.; Nakajima, T.; Xu, D.; Homma, K.; Kokubun, M. Genotypic variation in salinity tolerance and its association with nodulation and nitrogen uptake in soybean. Plant Prod. Sci. 2017, 20, 490-498. [CrossRef]

62. Di Gioia, F.; Tzortzakis, N.; Rouphael, Y.; Kyriacou, M.C.; Sampaio, S.L.; Ferreira, I.C.F.R.; Petropoulos, S.A. Grown to be blue-antioxidant properties and health effects of colored vegetables. Part II: Leafy, fruit, and other vegetables. Antioxidants 2020, 9, 97. [CrossRef]

63. Corrado, G.; Chiaiese, P.; Lucini, L.; Miras-Moreno, B.; Colla, G.; Rouphael, Y. Successive harvests affect yield, quality and metabolic profile of sweet basil (Ocimum basilicum L.). Agronomy 2020, 10, 830. [CrossRef]

64. Rouphael, Y.; Petropoulos, S.; Cardarelli, M.; Colla, G. Salinity as eustressor for enhancing quality of vegetables. Sci. Hortic. 2018, 234, 361-369. [CrossRef]

65. Carillo, P.; Woodrow, P.; Raimondi, G.; El-Nakhel, C.; Pannico, A.; Kyriacou, M.C.; Colla, G.; Mori, M.; Giordano, M.; De Pascale, S.; et al. Omeprazole promotes chloride exclusion and induces salt tolerance in greenhouse basil. Agronomy 2019, 9, 355. [CrossRef]

66. Diatloff, E.; Roberts, M.; Sanders, D.; Roberts, S.K. Characterization of anion channels in the plasma membrane of arabidopsis epidermal root cells and the identification of a citrate-permeable channel induced by phosphate starvation. Plant Physiol. 2004, 136, 4136-4149. [CrossRef] [PubMed]

67. Adeyemi, O.; Grove, I.; Peets, S.; Domun, Y.; Norton, T. Dynamic modelling of lettuce transpiration for water status monitoring. Comput. Electron. Agric. 2018, 155, 50-57. Available online: https:/ / core.ac.uk/download/pdf/288197019.pdf (accessed on 29 March 2021). [CrossRef] 